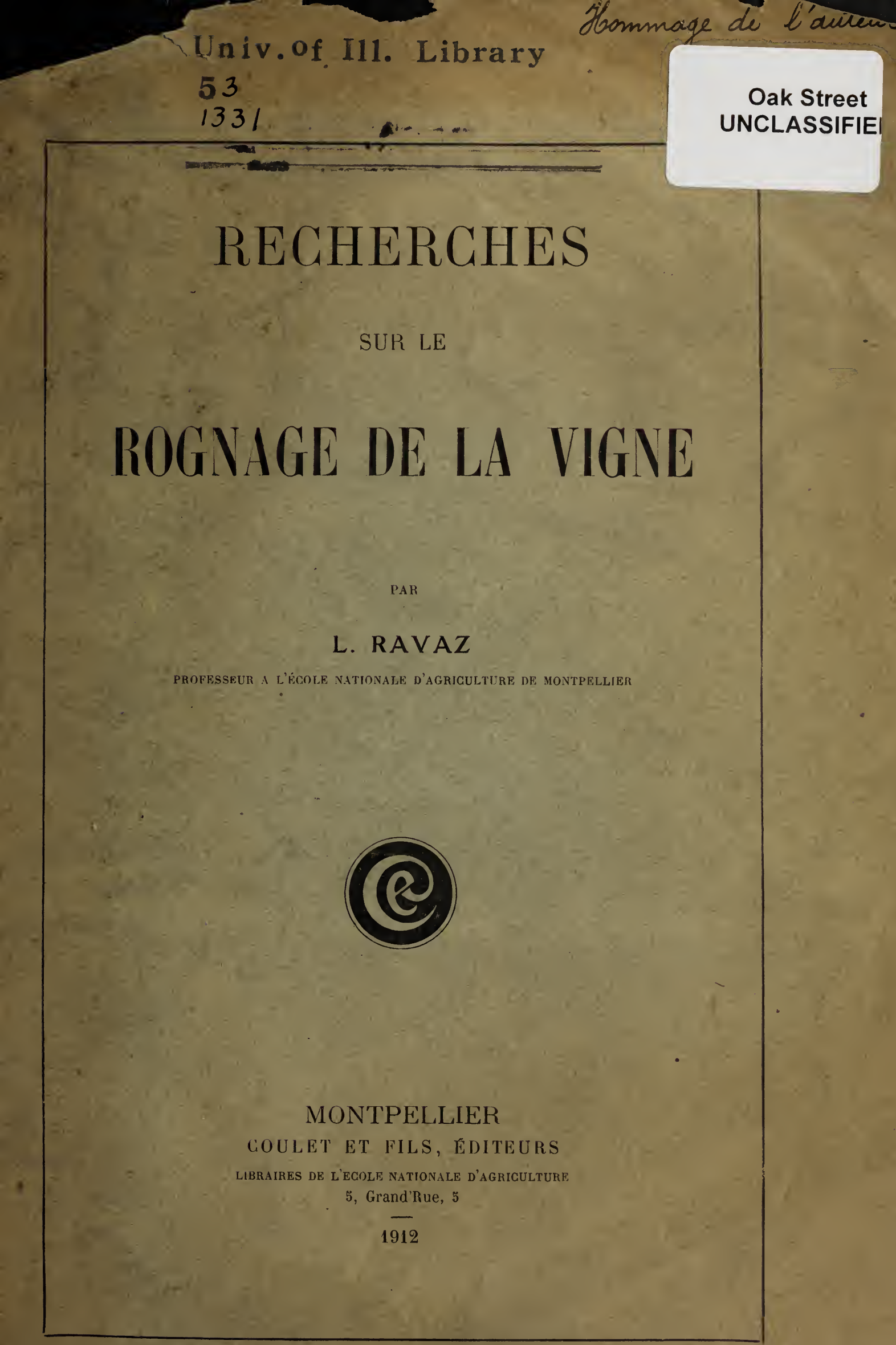





\section{RECHERCHES}

SUR LE

\section{ROGNAGE DE LA VIGNE}





\section{RECHERCHES}

SUR LE

\section{R0GNAGE DE LA VIGNE}

PAR

L. RAVAZ

PROFESSEUR A L'ÉCOLE NATIONALE D'AGRICULTURE DE MONTPELLIER

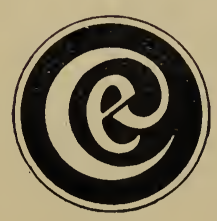

MONTPELLIER

COULET ET FILS, EDITEURS

LIBRAIRES DE L'ECOLE NATIONALE D'AGRICULTURE

5, Grand'Rue, 5 



\title{
RECHERCHES \\ SUR LE ROGNAGE DE LA VIGNE
}

\author{
Par M. L. RAVAZ \\ Professeur à l'École nationale d'Agriculture de Montpellier
}

Historique. - Comme la plupart des opérations culturales viticoles, le rognage est pratiqué depuis fort longtemps. D’après Savastano, "Théophraste, dans son Histoire desplantes, appelle ") le rognage $\beta \lambda_{\alpha \sigma \tau o \lambda o \gamma\llcorner\alpha}$ de $\beta \lambda_{\alpha \sigma \tau}$, (bourgeon). Dans le livre III, » chapitre XIV, paragraphe I dudit ouvrage, se trouve le passage ») suivant que je traduis textuelle» ment : Quand la vigne bour»geonne, il faut enlever tous les sarments qui ne portent pas » de fruits. Des fructifères, on enlève la partie au-dessus des "fleurs, grappes, afin que la vigne ne se développe pas en sar") ments et que ce qui reste en aliments, soit accumulé dans les "fleurs et dans les grappes.

»Et ailleurs, livre III, chapitre XVI, paragraphe I, il dit qu'après l'opération de la taille vient par importance celle du rognage, laquelle est faite avant la formation du fruit(1) ).

Sous le nom de pampinatio, les latins désignent toutes les opérations de la taille en vert. Leurs agronomes conseillent, en en montrant toute l'importance, l'ébourgeonnage,l'effeuillage, etc.. mais ils citent rarement le rognage, sauf Columelle qui dit : “ qu'il faut briser du doigt l'extrémité des sarments».

Faut-il en conclure que le rognage n'était en usage que dans

(1) L. Savastano. Studio della cimatura della vite, in Le Stozioni sperimentalie agrarie. Roma 1888. 
quelques localités ou dans certaines conditions de milieu et de cépage? Il est probable que, pas plus qu'actuellement, il n'était alors une pratique générale. Mais, dans un tel sujet, on peut tout conjecturer. Et il ne faut pas oublier que lesécrivains insistent plus volontiers sur les pratiques nouvelles ou peu con nues, qui ont comme un certain attrait de la nouveauté que sur celles qui sont bien établies et connues de tous.

En Italie. - Actuellement, le rognage est pratiqué comme autrefois au pays de Théophraste. Il l'est aussi en Italie. Dans la région Vésuvienne, dit Savastano, «on pratique en mai la taille en vert avant que les grappes fleurissent. On enlève tous les rameaux sans grappes des sarments à fruit recourbés, les rameaux munis de grappes sont pincés à deux feuilles au-dessus de la dernière. Les rameaux à bois se réduisent à la longuer que le viticulteur croit que la vignepeut porter. Enfin, on enlève les gourmands du tronc.

"Il n’y a aucune exagération à affirmer qu'avec la taille de mai on enlève à la vigne les $3 / 4$ de son feuillage. Une vingtaine de jours après ce premier raquage on en fait un second, en enlevant les rameaux secondaires, les gourmands; et quelquefois mème on pratique une troisième opération ».

Il est aussi plus ou moins en usage dans les vignobles de l'Allemagne, de la Hongrie, des còtes méditerranéennes pour certains cépages, de l'Espagne.

En Suisse. - En suisse il est pratiqué avec beaucoup de soins, notamment dans le canton de Vaud. Tous les rameaux réservés après un ébourgeonnage sévère sont palissés à un échalas de $1^{\mathrm{m}}, 30$ et rognés à hauteur de son sommet, c'est-àdire à 10 ou 12 feuilles au-dessus de la base. Ce rognage, qui est fait avant la floraison, fait développer en rameaux, soit des entı-cœurs (rebiots ou entrefeuilles), soit les yeux les plus élevés, qui doivent ètre supprimés - sur leur empàtement ou à une feuille au-dessus - au fur et à mesure de leur apparition. Il en résulte le développement de nouvelles ramifications qui sont 
supprimées ou réduites comme les premières ; de mème pour celles qui apparaitraient plus tard. Le cep et ses raisins sont donc alimentés dès le premier rognage par les 10 ou 12 feuilles primordiales de chaque rameau conservé. Cette pratique a pour objet, dit Jacques Boujour, «de favoriser la végétation, d'éviter un épuisement inutile du cep et surtout de faire augmenter et prospérer le raisin. Les jeunes vignes sont soumises à cette opération cornme les vignes «en force ». Lorsque la végétation est très active, elle hàte quelquefois trop le développement des yeux. Il n'est point pratiqué dans le Valais.

En France. - En France, le rognage n'est pas en usage dans tous les vignobles. Il n'est pas appliqué au Gamay du Beaujolais, de la Loire et de la Haute-Loire, du Màconnais, de la Bourgogne, plaine et arrière còte, de Bar-sur-Aube, du Puy-de-Dòme, etc., sauf sur les ceps très vigoureux; partout où il est cultivé, ou presque partout, ce cépage conserve ses rameaux intacts. Est-ce parce qu'ils sont relativement courts et ordinairement dressés ? G'est probable. Notons aussi qu'il est si fertile, qu'il n'y a lieu de mettre en œuvre les moyens qui assurent une bonne fructification que sous un climat défavorable, tel celui des environs de Paris. - La Syrah et le Viognier de Còte-Rotie, ne sont pas non plus rognés, et l'on ne rogne ni à Marzy, dans la Nièvre, ni à Bourges, dans le Cher, où «les pampres sont cependant très vigoureux et dépassent souvent les échalas de $\mathbf{1}^{\mathrm{m}}, 33$ et retombent en courbes que beaucoup de viticulteurs se plaisent à réunir et à attacher en arcades par 2 ceps » (1); ni les vignes du Bas-Rhin conduites en Kammerbau; ni dans la Sarthe, l'Ile-etVilaine, l'Anjou et le Saumurois, le Nantais, la Touraine, la Vendée, les Deux-Sèvres, les Charentes, la Vienne, la HauteVienne. Si elle n'y est pas inconnue, cette opération n'est pas en usage dans les vignobles de l'Ouest et du Nord-Ouest de la France. Elle ne l'est pas non plus dans toute la région méridio- 
nale de la France: Provence, Languedoc, Roussillon, HauteGaronne, Pyrénées, Landes, Gers, Lot, Lot-et-Garonne, etc., sauf pour certains cépages, dont elle a pour but d'assurer la fructification.

Le rognage est exceptionnellement pratiqué dans l'Isère, les Savoies, l'Ain, le Jura, où l'on «mouche» les rameaux de l'extrémité de la «courgée » ou long bois.

Mais il devient une pratique régulière à Pouilly, où les trois derniers rameaux de la " queue » (long bois) du Chardonnet, sont « mouchés » à 2 feuilles au moment de l'accolage; dans la Bourgogne, où Pinots et Chardonnay sont rognés au mois de juillet, quelques jours après la floraison, à hauteur de l'échalas. Le nombre de mérithalles enlevés est, par suite, variable. Vergnette Lamothe dit qu'après cette opération, il reste sur le cep environ 88 feuilles qui, sur leurs deux faces, donnent 140 décimètres carrés (in Le Vin). Elle est, dit Caumon-Bréon, « d'autant plus utile qu'elle ralentit la circulation de la sève au profit du fruit ». Quoi qu'il en soit, elle a pour conséquence de favoriserle développement des entre-cœurs ou rebiots, que l'on supprime le plus tòt possible, soit sur leur empàtement, soit à une ou deux feuilles au-dessus; on est ainsi amené, suivant la vigueur des ceps, à pratiquer 3,4 et mème 气̆ rognages successifs.

On rogne aussi en juillet, c'est-à-dire peu après la fleur, puis en septembre les Pinots de Bar-sur-Aube; après la fleur et une autre fois après la sève d'aoùt, les Pinots, Gamay, etc., de Barsur-Seine, des Ricey, des environs de Troyes. "Dans l'Yonne, le rognage est une pratique régulière » : aux environs d'Auxerre, à Coulanges-la-Vineuse, à Chablis après l'accolage etaprès la fleur autant que possible. Des bourgeons anticipés se développent et sont ensuite enlevés ou rognés plus ou moins suivant la rapidité de leur développement, qui est en relation directe avec la température et l'humidité. Ce rognage, en arrètant momentanément la végétation, favorise la formation des grains du raisin d'abord, puis la maturité des fruits et du bois (Chappaz).

Dans l'Allier, le rognage a lieu en juillet et aoùt, avant la fleur dans quelques localités (Brousse) de la Nièvre; en juillet à la 
Charité. Dans le Sancerrois, les rameaux qui dépassent l'échalas sont rognées à sa hauteur lors du premier accolage, c'est-à-dire à la floraison, et on les rogne de nouveau à mesure qu'ils grandissent en mème temps qu'on supprime toutes les repousses pour laisser pénétrer l'air et le soleil ». Dans le Gàtinais et l'Orléanais, on pratique le rognage après la fleur, en juillet et à plusieurs reprises jusqu'à la vendange - pour nourrir le bétail dit J. Guyot - mais sans doute aussi pour une autre raison.

Dans le Haut-Rhin, on rogne à $2-4$ feuilles au-dessus des raisins les rameaux qui seront supprimés à la taille d'hiver, et plus tard en juillet-aoùt ceux qui doivent fournir les bois de remplacement. On opère de mème dans les vignobles dela Meurthe, des environs de Toul, de Pont à Mousson, de Thiancourt, dela Moselle où l'on supprime avant la fleur les vrilles, les entrefeuilles, les gourmands ; de la Haute-Marne, etc.

Dans toute cette région du Nord-Est, le premier rognage est pratiqué en 2 fois: il porte d'abord sur les rameaux des longs bois, qui sont raccourcis à 1, 2, 3 ou 4 feuilles au-dessus de la dernière grappe; il porte ensuite en juillet-aoùt sur les rameaux de remplacement.

En Champagne, le rognage est la règle. Au moment de l'accolage, on coupe les rameaux à hauteur de l'échalas s'ils sont vigoureux; plus bas s'ils sont faibles. Au fond, chaque rameau est réduit à 10 ou 12 feuilles. On a soin aussi d'enlever les entrecœurs ou de les raccourcir à une ou 2 feuilles au fur et à mesure qu'ils se développent. On est ainsi amené à faire $3,4,5$ rognages successifs suivant que la végétation est plus ou moins active. «Le rognage, dit M. Mazade, est une opération indispensable en Champagnẹ. C'est le régulateur essentiel de la végétation. Il permet de maintenir sur une surface donnée un très grand nombre de ceps de vigueur sensiblement égale ; d'assurer la maturité car lorsque le raisin va mùrir, il faut que le développement de la partie végétalive soit arrèté au bénéfice de la maturation ». Il est peut-ètre aussi indispensable pour qu'on puisse circuler autour du cep, travailler le sol, etc. ; et enfin il a d autres conséquences sur lesquelles nous reviendrons plus loin. 
Des vignobles de l'Ouest, le Médoc et les Graves, sont les seuls où le rognage soit régulièrement pratiqué. «A près la floraison, dit Boireau, on épampre : cette opération consiste à couper le bout des pousses des sarments; elle se fait une première fois vers la fin juin; on la renouvelle dans le mois d'aoùt, un peu avant que les raisins viennent à changer ; dans les années pluvieuses, on épampre plus qu'on ne le fait dans les années de sécheresse ».

" A l'époque de la floraison, dit C. Alibert, on épampre nos vignes en les taillant en brosse à la faveur d'un morceau de vieille faux adapté à un manche et que le vigneron dirige horizontalement. Le vigneron supprime les longueurs exagérées des sarments tant au-dessus qu'au dessous du billon. Nos habitudes sur ce point sont faciles à justlfier. L'épamprage, tèl que nous le pratiquons a pour effet: $1^{\circ}$ de dégager le billon d'une végétation exubérante et qui gènerait le passage desbœufs ; $2^{\circ}$ de permettre au cabat et à la courbe de fonctionner avec plus d'aisance et au laboureur de les diriger avec plus de sùreté; $3^{\circ}$ de laisser passer les rayons solaire dans le fourré trop épais du feuillage $4^{\circ}$ de prévenir la coulure.

Après la floraison, on épampre encore aveé légèreté dans les

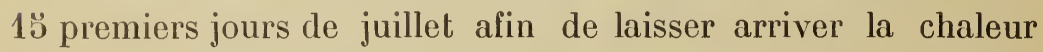
directe du soleil dans le billon et de rendre plus libre la voie que parcourent les bœufs... Enfin on épampre une 3 e fois au moment des vendanges, pour que les vendanges puissent avancer rapidement $)$.

Enfin, on rogne au niveau de l'échalas les rameaux de remplacement et à une feuille au-dessus de la dernière grappe dans le Petit vignoble de Cap Breton (Landes)..

C'est donc, si l'on excepte la Gironde, au Centre et au Nordest de la France que le rognage est régulièrement pratiqué.

Utilité - Il n'est donc pas pratiqué dans toutes les régions viticoles. D'un usage constant dans un vignoble, il peut ètre inconnu dans le vignoble voisin complanté des mèmes cépages, dans des sols et sous des climats semblables (Gironde). Son 
utilité est-elle donc contestable ? ou bien est-elle liée à certaines particularités culturales? C'est ce que nous allons étudier maintenant.

Opıntons - Olivier de Serres conseille le rognage pour éviter les effets du vent, qui casse les grappes et les pampres. "A cela, le remède est de rogner les cimes des jettons que verrés fort jetter en dehors, contraignant, par ce moyen, la vigne de se maintenir en ses limites » et il ajoute: "remède qui servira aussi au profit des raisins restant sur leurs appuis pour s'engrossir ».

Le comte Odart écrit: "Le rognage a, pour certaines vignes, une importance incontestable: ce sont celles en treilles labourées pour faciliter le passage des bètes de labour ; car pour les autres, cette opération ne me semble qu'un soin de propreté, et être principalement avantageuse au vigneron qui y trouve la nourriture de sa vache. Il n'est pas du tout prouvé qu'elle offre un avantage bien réel et bien sensible pour la récolte. Le rognage de la partie au-dessus de l'échalas est toutefois fort convenable dans les jeunes vignes qui poussent violemment parce que le sarment s'allongeant beaucoup, charge la tète, et lors des grands vents, cette charge ébranle l'échalas, et il est plus facilement renversé par eux avec le cep. Ce qui peut faire juger aussi de son peu d'importance, c'est qu'elle est abandonnée aux femmes. »

Chabert (in Rozier) dit que cette opération n'est utile (à Argenteuil) que dans les années où la vigne pousse avec trop d'énergie, elle empèche que les bourgeons ne s'accrochent les uns aux autres par leurs vrilles ; elle procure aussi un excellent fourrage, etc.

Cavoleau la considère comme nuisible. « Nous en avons dit assez, écrit-il, pour faire concevoir que la rognure est une opération vicicuse. En effet, en supprimant la portion des sarments qui s'élève au-dessus des échalas, on supprime une grande quantité de feuilles. Il n'est donc pas étonnant, comme l'a observé Rozier, et comme du reste, on peut l'observer partout, que la rognure épuise la vigne et nuise à la qualité de son fruit. » 
Dubreuil, au contraire, conseille le rognage "pour diminuer les chances de coulure des grappes, favoriser leur développement et éviter l'enchevètrement et la confusion de la végétation. »

Les opinions qui précèdent sont basées, pour la plupart, sur des faits d'observation, ou bien sont l'écho de l'opinion des praticiens. On doit évidemment en tenir compte. Toutefois, elles sont insuffisantes pour résoudre la question que nous étudions puisqu'elles sont contradictoires. L'expérimentation peut-elle nous donner des résultats plus probants?

Expériences. - De nombreuses expériences ont été faites en France et à l'étranger pour déterminer la valeur du rognage. Les résultats de quelques-unes, qui ont été faites en Italie, sont donnés dans le tableau suivant:

\section{Influence du rognage sur la maturation du raisin. - Composition des raisins}

\begin{tabular}{|c|c|c|c|c|c|c|}
\hline & \multirow{2}{*}{\multicolumn{2}{|c|}{$\frac{\text { Vignes témoin }}{\text { lucose } \% \text { Acidité } \%}$}} & \multicolumn{2}{|c|}{ Vignes rognées } & \multirow{2}{*}{\multicolumn{2}{|c|}{$\underbrace{\begin{array}{c}\text { Vignes rognées } \\
\text { plusieurs fois }\end{array}}_{\text {Gluc. } \% \text { Acid. } \%}$}} \\
\hline expérimentateurs & & & Glucose $\%$ & Acidité $\%$ & & \\
\hline Macagno. . & 17.540 & 13.200 & 14.600 & 14.000 & & \\
\hline Cerletti. . . . & 14.500 & 22.44 & 14.20 & 22.20 & & \\
\hline Soldani. & & & & & 19 & $7.7 \breve{5}$ \\
\hline - & 20.60 & 9.25 & 19 & 9 & 18.500 & \\
\hline Savastano. & 14.73 & 7.00 & 10.75 & 8.70 & ) & 》 \\
\hline
\end{tabular}

La conclusion de ces travaux est que le rognage est nuisible à la qualité du produit de la vigne et «d'autant plus, ajoute Savastano, que la vigne est plus vigoureuse ».

La production serait aussi réduite : car 100 grappes de vignes non écimées pèsent de 18 k. 800 à 29 kilogrammes et 100 grappes de vignes écimées de 4 k. 400 à 22 kilogrammes. La réduction suivant les variétés, dépasse parfois 50 o/o ; elle est aussi plus forte pour les vignes vigoureuses que pour les vignes faibles (1). 
Müller-Thurgau a étudié, pendant plusieurs années, les effets du rognagne sur le Riesling cultivé dans le Rhingau ; il a obtenu les résultats suivants :

\begin{tabular}{ccccc}
$\begin{array}{c}\text { Numéros } \\
\text { des } \\
\text { parcelles }\end{array}$ & $\begin{array}{c}\text { Nombre de feuilles } \\
\text { conservées au dessus } \\
\text { des grappes }\end{array}$ & \multicolumn{3}{c}{ Richesse en sucre pour 100} \\
\cline { 3 - 5 }- & $\begin{array}{c}\text { 2 feuilles } \\
1\end{array}$ & 13.95 & 11.905 & 16.73 \\
2 & 4 & 14.40 & 12.195 & 16.95 \\
3 & 6 & 14.65 & 12.346 & 17.36 \\
4 & Témoin & 15.15 & 12.422 & 17.70
\end{tabular}

La teneur en sucre est en raison inverse du nombre de feuilles enlevées. Et il en est de mème de la quantité de raisin produite, ainsi que le montre le tableau suivant :

\begin{tabular}{|c|c|c|c|c|}
\hline $\begin{array}{c}\text { Numéros des } \\
\text { parcelles } \\
-\end{array}$ & $\begin{array}{l}\text { Nature du } \\
\text { rognage } \\
-\end{array}$ & $\frac{1882}{\text { kil. }}$ & $\begin{array}{l}\text { Production en } \\
1883 \\
\overline{\text { kil. }}\end{array}$ & $\frac{1884}{\text { kil. }}$ \\
\hline 1 & à 2 feuilles & 14.96 & 18.30 & 3อั. ธ3 \\
\hline 2 & à 4 & 18.17 & 23.00 & 46.28 \\
\hline 3 & à 6 & 17.06 & 23.00 & อั1.64. \\
\hline 4 & Témoin & 20.90 & 30.40 & 49.48 \\
\hline
\end{tabular}

Quantité et qualité sont donc ici diminuées.

En France, M. Duclou a étudié les effets de cette opération sur la vigne et le vin de la Gironde. Les voici :

\begin{tabular}{lll} 
& \multicolumn{1}{c}{$\begin{array}{c}\text { Témoin } \\
\text { Densité }\end{array}$} & $\begin{array}{c}\text { Vignes rognées } \\
\text { Densitê }\end{array}$ \\
Malbec $\ldots \ldots \ldots \ldots \ldots$ & $13^{\circ} \bar{B}$ Baumé & $12^{\circ} 25$ \\
Merlot $\ldots \ldots \ldots \ldots \ldots$ & $13^{\circ} 75$ & $13^{\circ} 60$ \\
Cabernet $\ldots \ldots \ldots \ldots$. & $14^{\circ}$ & $12^{\circ} 75$
\end{tabular}

Ici encore, le rognage aboutit à la diminution de la qualité du moùt.

M. Rivière a étudié les effets du rognage sur le Chasselas; ils sont indiqués dans le tableau suivant: 
Rapport entre le nombre de feuilles conservées au-dessus des grappes sur les bourgeons fertiles et la richesse en sucre des grains de raisin. (Chasselas doré).

\begin{tabular}{|c|c|c|c|c|c|c|c|}
\hline $\begin{array}{c}0 \\
\text { feuille } \\
-\end{array}$ & $\begin{array}{c}1 \\
\text { feuille } \\
-\end{array}$ & $\begin{array}{c}2 \\
\text { feuilles } \\
-\end{array}$ & $\begin{array}{c}3 \\
\text { feuilles } \\
-\end{array}$ & $\begin{array}{c}4 \\
\text { feuilles } \\
-\end{array}$ & $\begin{array}{c}5 \\
\text { feuilles } \\
-\end{array}$ & $\begin{array}{c}6 \\
\text { feuilles } \\
-\end{array}$ & $\begin{array}{c}7 \\
\text { feuilles } \\
-\end{array}$ \\
\hline \multicolumn{8}{|c|}{ Sucre (par litre de moût) } \\
\hline $\begin{array}{c}\text { gr. } \\
159,00\end{array}$ & $\begin{array}{c}\text { gr. } \\
165,20\end{array}$ & 185,20 & $\begin{array}{c}\text { gr. } \\
191,60\end{array}$ & $\underset{198,40}{\mathrm{gr}}$ & $\underset{205,00}{\text { gr. }}$ & $\begin{array}{c}\text { gr. } \\
205,00\end{array}$ & $\begin{array}{c}\text { gr. } \\
205,00\end{array}$ \\
\hline \multicolumn{8}{|c|}{ Acidilé (exprimée en $\mathrm{SO}^{4} H^{2}$ par litre de moût) } \\
\hline 3,95 & 3,90 & 3,85 & 3,80 & 3,68 & 3,65 & 3,65 & 3,65 \\
\hline
\end{tabular}

«De la lecture de ce tableau, il ressort une fois de plus, dit l'auteur, que le pincement des bourgeons fertiles du Chasselas doré exerce une action plus ou moins favorable sur la composition chimique du jus contenu dans les grains de raisin de cette variété, selon qu'il est pratiqué plus ou moins sévèrement.

" En effet, suivant que cette opération a été exécutée immédiatement au-dessus de la seconde grappe d'un bourgeon fertile ou qu'elle a été effectuée au-dessus de 1, 2, 3, 4, 5, 6, 7 feuilles situées au-dessus de la seconde grappe d'un bourgeon de mème nature, on constate que la proportion de sucre contenue dans le moùt du raisin, s'accroît non pas indéfiniment au fur et à mesure que le nombre de feuilles augmente, mais jusqu'à une certaine limite qui, dans la circonstance, correspond exactement à la cinquième feuille puisque, à partir de cette cinquième feuille, la proportion de sucre ne s'accroìt plus dans le moùt du Chasselas doré.

( De 1599 grammes par litre de moùt, quand on ne conserve aucune feuille au-dessus des grappes des bourgeons fertiles, cette quantité s'élève jusqu à 203 grammes lorsqu'on en laisse subsister 5,6 ou 7 .

“Quant à l'acidité, on constate un phénomène absolument inverse; sa proportion diminue progressivement dans le moût au fur et à mesure que le nombre de feuilles augmente au-dessus des grappes. De 3,93 elle s'abaisse à 3,63 par litre de moùt suivant que le pincement a été fait immédiatement au-dessus des 
grappes, ou que l'on a conservé 5 , 6 ou 7 feuilles au-dessus de ces mèmes grappes Comme nous l'avons fait observer relativement au sucre, la proportion d'acidité, dans le jus du Chasselas, ne varie plus à partir de la 马̆e feuille ».

On peut conclure de ce travail que si le rognage à $\breve{b}$ feuilles et au delà ne diminue pas la qualité, il ne l'améliore jamais. On ne saisit pas l'utilité de cette opération.

L'accroissement de la qualité consécutif au rognage a rarement été constaté. M. Cazeaux-Cazalet le considère cependant comme la règle pour le rognage tardif, fait au moment où la végé. tation se ralentit d'une manière marquée, c'est-à-dire un peu avant la véraison « En comparaison avec des raisins provenant de ceps non rognés, j'ai constaté, dit-il, en 1894, que des raisins blancs de Sémillion, cueillis sur des ceps voisins rognés, avaient de 0,5 à 2 o/o de sucre en plus; en 1907 , j'ai trouvé de 0,3 à 1,5 o/o en plus sur tous les cépages rognés, rouges et blancs, et dans les terrains les plus divers ». Il favorise aussi le grossissement et l'aoùtement des sarments; “ le meilleur aoùtement des vignes rognées a été constaté pendant plusieurs années au microscope en recherchant l'état de la lignification et l'amidon; on l'a reconnu aussi à la dureté des bois rognés. Nous sommes donc fondés à dire que dans tous les climats, sur tous les cépages, le rognage est une bonne opération à la condition essentielle de la faire au moment de l'arrêt de végétation qui suit la floraison, afin d'éviter des rejets et à la hauteur suffisante pour couper sur une partie " herbacée ».

Le gain signalé par M. Cazeaux-Cazalet n'est pas très considérable : il indique toutefois que la qualité a plutòt gagné que perdu à l'opération.

MM. Pacottet et Jeannin ont pratiqué le rognage en coupant " les jeunes pousses avec l'ongle et sur les nœuds, successivement à $0,1,2,3$, 4 feuilles au-dessus du dernier raisin. L'opération a été faite quelque temps avant la floraison; puis ils ont laissé croitre le dernier bourgeon axillaire dont ils ont, à nouveau, pincé la tige, suivant les sarments à $4, \check{5}, 6,7$ et $\delta$ feuilles au-dessus du dernier raisin. Chaque pampre a été maintenu dans cet état jusqu'à la maturité ». Résultats : 
Témoin pris sur des ceps conduits en golvelets..........

Branches écimées à 0 feuille rognées à 4 feuilles .... ..

Branches écimées à 2 feuilles rognées à 4 feuilles........

Branches écimées à 0 feuille rognées à 6 feuilles.......

Branches écimées à 2 feuilles rognées à 8 feuilles.........

Branches écimées à 4 feuilles maintenues à 4 feuilles....

Branches rognées à 6 feuilles.

Branches rognées à 10 feuilles.

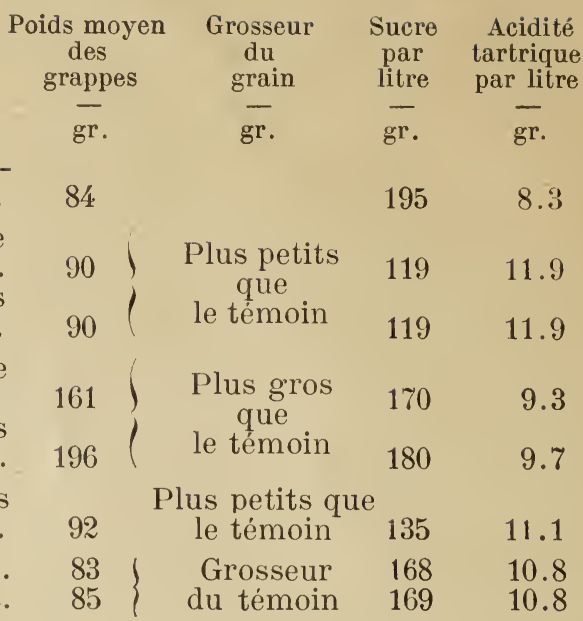

J'ai fait également, pendant plusieurs années, à l'École d'agriculture de Montpellier, des recherches sur ce point. Elles ont porté sur plusieurs cépages français.

1. Dans la vigne Claparède, le rognage a été pratiqué aux époques indiquées ci-après. Il a toujours consisté dans la suppression des extrémités en voie de croissance, c'est-à-dire des 7 ou 8 mérithalles terminaux.

\section{Expérience I. - Aramon}

Les souches qui ont servi à cette expérience sont de même àge, jeunes et plutòt puissantes. Je ne donne pas le poids de sarments, car il est toujours plus faible sur les vignes rognées que sur les témoins. Il est donc impossible de tenir compte ici de $\frac{\mathrm{F}}{\mathrm{V}}$.

A défaut de ce rapport, il y a lieu d'envisager le poids de la récolte. Il subit des variations qui sont peu importantes. Les rognages, quels qu'ils soient, n'ont pas modifié sensiblement la production. L'Aramon, dans les conditions de nos expériences, n'en a ni bénéficié ni souffert. Dans d'autres sols et sur des sujets puissants, favorisant la coulure, il pourrait en ètre autrement. 
LE ROGNAGE DE LA VIGNE

\begin{tabular}{|c|c|c|c|c|c|c|c|}
\hline \multirow{3}{*}{ 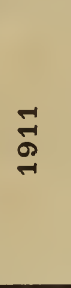 } & 起 む & क़ & ळี & 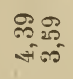 & $\begin{array}{l}\text { 墭 } \\
\text { का }\end{array}$ & $\begin{array}{l}\text { ले } \\
\text { की }\end{array}$ & 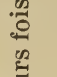 \\
\hline & 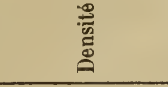 & 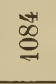 & $\stackrel{R}{\circ}$ & 융도 & ĩ & 5 & $\frac{\mathscr{2}}{\stackrel{2}{3}}$ \\
\hline & 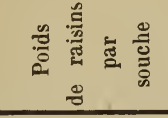 & 20. & $\begin{array}{l}\text { 尺 } \\
\text { oi }\end{array}$ & 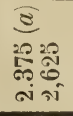 & $\begin{array}{l}\text { Aึ } \\
\text { oi } \\
\text { जi }\end{array}$ & $\begin{array}{l}\text { तิ } \\
\text { oै } \\
\text { oi }\end{array}$ & 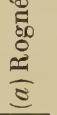 \\
\hline \multirow{3}{*}{$\stackrel{0}{\stackrel{-}{\sigma}}$} & 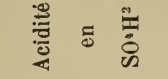 & क्ष & $\begin{array}{l}\infty \\
0 \\
0\end{array}$ & \& & $\begin{array}{l}80 \\
0\end{array}$ & $\begin{array}{l}\mathscr{\infty} \\
0 \\
0\end{array}$ & \\
\hline & 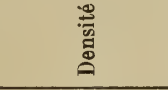 & $\underset{8}{\stackrel{8}{8}}$ & $\stackrel{0}{0}$ & $\overbrace{0}^{28}$ & $\underset{-1}{E}$ & $\underset{\mathscr{E}}{\mathscr{E}}$ & \\
\hline & 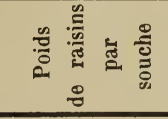 & $\stackrel{\overbrace{}}{8}$ & สิ & $\begin{array}{l}\overrightarrow{80} \\
20\end{array}$ & \begin{tabular}{l}
$\infty$ \\
30 \\
\multirow{2}{*}{}
\end{tabular} & क्ष & \\
\hline \multirow{3}{*}{ ஓ } & 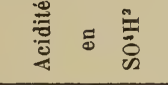 & $\stackrel{2}{0}$ & $\begin{array}{l}5 \\
60 \\
0\end{array}$ & $\vec{E}_{20}$ & $\begin{array}{l}8 \\
0 \\
0\end{array}$ & $\begin{array}{l}\text { 구 } \\
=0\end{array}$ & \\
\hline & 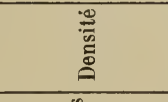 & $\begin{array}{l}20 \\
8 \\
8\end{array}$ & $\frac{20}{20}$ & $\begin{array}{l}20 \\
50 \\
20 \\
=\end{array}$ & $\begin{array}{l}20 \\
20 \\
20 \\
20 \\
\end{array}$ & 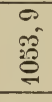 & \\
\hline & 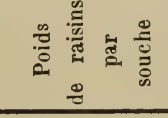 & \begin{tabular}{l} 
जै \\
20 \\
\multirow{4}{*}{}
\end{tabular} & i̊ & 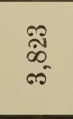 & $\stackrel{28}{2}$ & $\stackrel{20}{=}$ & \\
\hline \multirow{3}{*}{$\begin{array}{l}\infty \\
0 \\
0 \\
2 \\
\sim-1\end{array}$} & 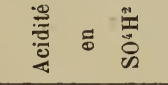 & $\begin{array}{l}5 \\
20\end{array}$ & $\stackrel{8}{\&}$ & $\begin{array}{l}28 \\
20 \\
20\end{array}$ & $\stackrel{R}{i}$ & 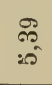 & \\
\hline & 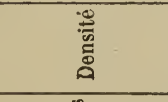 & है & है & $\begin{array}{l}20 \\
8 \\
8\end{array}$ & $\begin{array}{l}\pi \\
\infty \\
\&\end{array}$ & $\stackrel{5}{E}$ & \\
\hline & 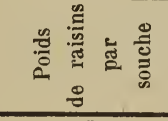 & $\stackrel{\vec{s}}{+}$ & ज्ञ & $\begin{array}{l}\text { बiे } \\
\text { wh } \\
\text { ثt }\end{array}$ & ثै & $\overrightarrow{5}$ & \\
\hline \multirow{2}{*}{ 5 } & 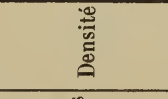 & 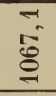 & है & $\overline{\hat{\theta}}$ & $\vec{E}$ & $\vec{~}$ & \\
\hline & 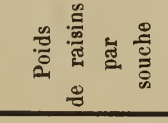 & $\stackrel{8}{=}$ & $\stackrel{?}{\stackrel{2}{*}}$ & 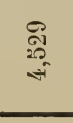 & \begin{tabular}{l} 
के \\
20 \\
\multirow{4}{*}{+}
\end{tabular} & 옹 & \\
\hline 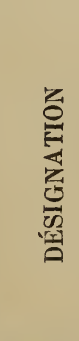 & 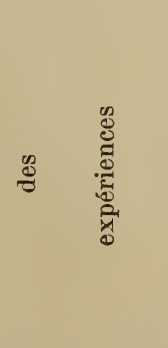 & 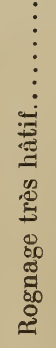 & 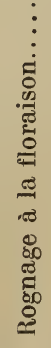 & 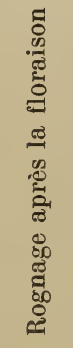 & 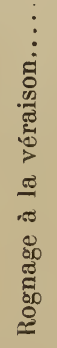 & $\begin{array}{c}\vdots \\
\vdots \\
\vdots \\
\vdots \\
\vdots \\
\vdots \\
\text { है }\end{array}$ & \\
\hline
\end{tabular}


Le rang rogné hàtivement a eu, seulement en 1911, une production déficitaíre plutòt sensible. Plusieurs causes ont pu entrer, ici, en jeu. La principale est, probablement, le Courtnoué qui s'est déclaré avec intensité sur ce rang. Le Court-noué serait-il en relation avec le rognage?

Quant à la qualité, elle parait être étroitement liée à la production ; elle suit presque constamment les variations de celle-ci. Cependant les rangs rognés portent des grappes quelquefois un peu plus riches en sucre que celles des témoins; le rang rogné de bonne heure est le moins modifié à ce point de vue.

En somme, la fertilité de l'Aramon et la qualité de ses produits sont peu modifiés par le rognage. Pourquoi? Probablement parce qu'il est un cépage qui ne coule pas et qui porte de gros grains. Supposant mème qu'il coule, l'augmentation du volume des grains restants compense au moins en partie, l'augmentation de leur nombre sur les pieds rognés. Et quant à la qualité, on peut remarquer que le nombre de feuilles enlevées est très faible par rapport au poids de la récolte. Leursuppression a sur chaque grappe une influence nécessairement très réduite.

\section{Expérience II. - Clairette}

Sur la Clairette, il en est autrement. En 1904, un rang pincé a donné 68 lilogs de raisins et le témoin $29 \mathrm{~kg}$.

L'augmentation de la production est de $124 \mathrm{o} / \mathrm{o}$; la qualité a été diminuée.

\section{Expérience III. - Pinot noir. 1909}

Le P̈̈not noir est généralement soumis au rognage, soit de bonne heure, soit tardivement. Il était intéressant d'étudier les conséqnences de cette opération sur ce cépage.

\begin{tabular}{|c|c|c|c|c|c|c|}
\hline $\begin{array}{l}\text { Numéros } \\
\text { des } \\
\text { parcelles }\end{array}$ & $\begin{array}{r}\mathrm{Na} \\
\mathrm{d} \\
\mathrm{rog}\end{array}$ & $\begin{array}{l}\text { ure } \\
\text { u } \\
\text { lage }\end{array}$ & $\begin{array}{l}\text { Poids de } \\
\text { raisins } \\
\text { par souche }\end{array}$ & $\begin{array}{l}\text { Augmentation } \\
\text { de la } \\
\text { production }\end{array}$ & Densité & $\begin{array}{l}\text { Acidité } \\
\text { en SO}{ }^{4} \mathrm{H}^{2}\end{array}$ \\
\hline 1 & rogné & 1 fois & 0,887 & $55 \%$ & 1086 & 5,47 \\
\hline 2 & - & 2 fois & 0,806 & $48^{\circ} /{ }^{\circ}$ & 1085 & 6,04 \\
\hline 3 & - & ๖ fois & 0,859 & $49 \%$ & 1084 & 6,28 \\
\hline 4 & Témoi & & $0, \breve{578}$ & 》 & 1093 & 6,39 \\
\hline
\end{tabular}


L'augmentation de la production est considérable et atteint 50 o/o. La diminution de la qualité est nette et importante: elle atteint 1 degrè 4.

$$
\text { Expérience IV. - Pinot noir }
$$

\begin{tabular}{|c|c|c|c|c|c|c|c|}
\hline $\begin{array}{l}\text { Nameros } \\
\text { des } \\
\text { parcelles } \\
-\end{array}$ & $\begin{array}{c}\text { Nature } \\
\text { du } \\
\text { rognage } \\
-\end{array}$ & $\begin{array}{l}\text { Poids de } \\
\text { raisins par } \\
\text { souche } \\
-\end{array}$ & $\frac{\mathrm{F}}{\mathrm{V}}$ & $\begin{array}{l}\text { Augmentation } \\
\text { de la } \\
\text { production } \\
\mathrm{k} .\end{array}$ & Densite & $\begin{array}{l}\text { Acidite } \\
\text { en } \mathrm{SO}^{0} \mathrm{H}^{2}\end{array}$ & Observations \\
\hline 1 & rogné 4 mai & 1,048 & 4,94 & $71 \%$ & 1083,7 & 5,73 & $\begin{array}{l}\text { Grappes compactes, } \\
\text { moût peu rosé. }\end{array}$ \\
\hline $1^{\text {bes }}$ & Témoin.... & 0,611 & $1, \ddot{2} 2$ & & 1093,7 & 5,73 & $\begin{array}{l}\text { Grap. làches, moût } \\
\text { plus rosé. }\end{array}$ \\
\hline 2 & & 0,460 & 4,29 & $42 \%$ & 1083,7 & 5,67 & $\begin{array}{l}\text { Belles grappes, com- } \\
\text { pactes; moût peu } \\
\text { coloré. }\end{array}$ \\
\hline $2^{\text {bst }}$ & Témoin.... & 0,280 & 0.417 & & 1087,7 & 5,70 & $\begin{array}{l}\text { Grappes lâches } \\
\text { moùt coloré. }\end{array}$ \\
\hline 3 & & 0,532 & 2,33 & $44 \%$ & 1083 & 5,56 & $\begin{array}{l}\text { Grappes compactes; } \\
\text { moût peu coloré. }\end{array}$ \\
\hline $3^{\text {bis }}$ & Témoin.... & 0,382 & 1,20 & & 1092,1 & 5,16 & $\begin{array}{l}\text { Grappes lâches, } \\
\text { grains petits ; } \\
\text { moût rosé. }\end{array}$ \\
\hline 4 & & 0,725 & 4,21 & $38 \%$ & 1077,1 & 6,37 & \\
\hline $4^{\text {bis }}$ & Témoin.... & 0,520 & 2,29 & & 1088 & 5,68 & \\
\hline
\end{tabular}

Ici encore et dans quatre parcelles différentes, gain considérable de récolte, mais diminution importante de la qualité portant sur la teneur en sucre et sur la matière colorante. La qualité paraît ici encore liée aux valeurs de $\frac{F}{V}$, c'est-à-dire à la production. Nous verrons plus loin si ce facteur doit être seul retenu

Expérience IVbis. Pinot noir IM. 1909

\begin{tabular}{|c|c|c|c|c|c|}
\hline $\begin{array}{l}\text { Numéros } \\
\text { des } \\
\text { parcelles } \\
\text { - }\end{array}$ & $\begin{array}{c}\text { Nature } \\
\text { du } \\
\text { rognage }\end{array}$ & $\begin{array}{l}\text { Production } \\
\text { par } \\
\text { souche } \\
-\end{array}$ & Densité & $\begin{array}{l}\text { Acidité } \\
\text { en } \mathrm{SO}^{4} \mathrm{H}^{2}\end{array}$ & Observations \\
\hline 1 & Rogné. & 0,572 & 1085 & ร้,68 & \\
\hline 2 & 'Témoin. & 0,447 & 1087 & 6,03 & gnes très touffues \\
\hline
\end{tabular}

Résultats de mème sens que les précédents, quoique peu marqués. La vigne d'expériences, vigoureuse, est extrêmement touffue, avec de longs sarments se recouvrant. Il semble que cet état est presque aussi défavorable à la qualité que le rognage. 


\section{Expérience V. Pinot noir B. G. 1910}

\begin{tabular}{|c|c|}
\hline $\begin{array}{l}\text { Numéros } \\
\text { des } \\
\text { parcelles }\end{array}$ & $\begin{array}{c}\text { Nature et époque } \\
\text { du } \\
\text { rognage }\end{array}$ \\
\hline$\overline{1}$ & res successifs \\
\hline & $\begin{array}{l}\text { pour supprimer } \\
\text { partie en voie de } \\
\text { croissance...... }\end{array}$ \\
\hline
\end{tabular}

21 seul rognage....

3 Rognage après la floraison ......

4 Rognage à la véraison ...........

๖ Rogné au moment de l'arrèt de la végétation ......

b Témoin .........

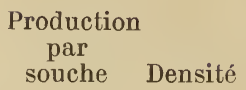

$0^{k} 452 \quad 1086$

$0500 \quad 1084,8$
Acidité

en $\mathrm{S}^{4} \mathrm{H}^{2}$ Observations

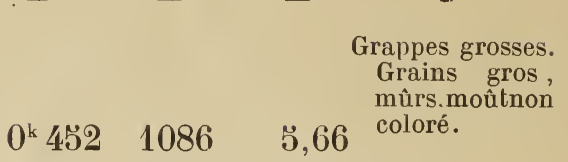

Grappes grosses. $\breve{\jmath}, 66$

Grains moins $6, \breve{1}$ gros et moins mûrs que 1.

Grainsplus petits que 2 , non $0411 \quad 1091,9 \quad 5$ כ, כ̌4 mûrs. Moût légèrement rosé.

Grappes petites. Grains petits. $03331093 \quad 5 ̋, 42$ Moût lègèrement rosé.

Vigne normale. Moût légère0307 1094,7 כ̋,76 ment rosé.

0318 1088,4 כ̌,つ̆4 $\begin{gathered}\text { Grappes compac- } \\ \text { tes. Grains pe- }\end{gathered}$ tits, bien mûrs.

La production est, ici encore, en relation étroite avec le rognage. Le rognage hàtif des parcelles 1, 2, 3 l'accroit; elle

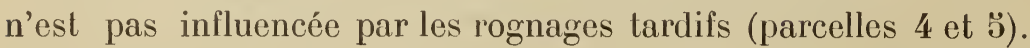
C'est donc, lorsqu'elle est pratiquée avant, pendant ou un peu après la floraison, que cette opération a été utile.

En ce qui concerne la qualité, le témoin a été, ici, défaillant; c'est qu'il s'est trouvé placé à l'ombre d'un arbre et que sa végétation s'est prolongée très tard. La parcelle 2 semble aussi faire exception : c'est que sa production est la plus élevée. Ces explications acceptées, il apparaìt que la qualité est d'autant plus diminuée que le rognage est fait plus tôt et répété plus souvent. 
Expérience VI. - Pinot noir. B. P. 1910

\begin{tabular}{|c|c|}
\hline $\begin{array}{l}\text { Numéros } \\
\text { des } \\
\text { parcelles }\end{array}$ & $\begin{array}{c}\text { Nature et époque } \\
\text { du } \\
\text { rognage }\end{array}$ \\
\hline 1 & $\begin{array}{l}\text { Rogné successive- } \\
\text { ment pour sup- } \\
\text { primer parties } \\
\text { en voie decrois- }\end{array}$ \\
\hline
\end{tabular}

21 seul rognage...

3 Rogné après la floraison........

4 Rogné à la véraison ..........

ร Rogné à l'arrêt de la végétation. .

6 Témoin.........

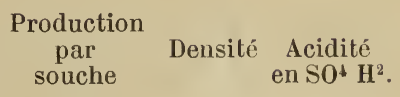

Observations Moût incolore.
Grappes compactes. Grains un peu petits, insuffisamınent mûrs.

Grappes compactes. Grains petits, insuffisamment mûrs. Moût incolore.

Grap. compac. Grains gros, bien mûrs. Moût incolore.

Grap. compac. Grains gros mûrs Moût un peu coloré.

Grappes petites Grains petits, mûrs Moût légèrement coloré.

Grappes petites 1096,9 6,05 Grainsnormeaux bien mûrs. Moût bien rosé.

Résultats concordants: quantité considérablement accrue par les rognages hâtifs, qualité diminuée.

\section{Expérience VII. - Pinot noir. M. 1910}

\begin{tabular}{cccccc}
$\begin{array}{c}\text { Numéros } \\
\text { des } \\
\text { parcelles }\end{array}$ & $\begin{array}{c}\text { Nature } \\
\text { du } \\
\text { rognage }\end{array}$ & $\begin{array}{c}\text { Production } \\
\text { par } \\
\text { souche }\end{array}$ & Densité & $\begin{array}{c}\text { Acidité } \\
\text { en S0 } \mathrm{H}^{2} .\end{array}$ & Observations \\
\hline 1 & Rogné ... & $0^{\mathrm{k}}, 420$ & - & - & - \\
2 & Témoin .. & $0^{\mathrm{k}}, 480$ & $1083, \breve{3}$ & & $\begin{array}{c}\text { Vignes extrême- } \\
\text { ment touffues }\end{array}$
\end{tabular}

Résultats inverses des précédents. Les vignes d'expériences sont très touffues. Le rognage, en facilitant l'éclairement des grappes, aurait-il favorisé la maturation? 
Expérience VIII. Pinot noir. H. R. 190.

\begin{tabular}{cccc}
$\begin{array}{c}\text { Numéros } \\
\text { des } \\
\text { parcelles }\end{array}$ & $\begin{array}{c}\text { Nature } \\
\text { du } \\
\text { rognage }\end{array}$ & $\begin{array}{c}\text { Production } \\
\text { par } \\
\text { souche }\end{array}$ & Densité \\
\hline 1 & Rogné.......... & $1^{k}, \overline{5} 00$ & - \\
2 & Témoin ......... & $1^{k}, 300$ & 1082,9 \\
& & &
\end{tabular}

Souches touffues. Augmentation de la production ; faible diminution de la qualité.

Expérience IX. - Pinot noir. B. G. 1911

\begin{tabular}{|c|c|c|c|c|c|}
\hline $\begin{array}{l}\text { Numéros } \\
\text { des } \\
\text { parcelles } \\
\text { - }\end{array}$ & $\begin{array}{c}\text { Nature et époque } \\
\text { du } \\
\text { rognage }\end{array}$ & $\begin{array}{l}\text { Production } \\
\text { par } \\
\text { souche } \\
\text { - }\end{array}$ & Densité & $\begin{array}{l}\text { Acidité } \\
\text { en SO'H' } \\
-\end{array}$ & Observations \\
\hline 1 & $\begin{array}{l}\text { Rognage continu. } \\
\text { Non effeuillé.... }\end{array}$ & $0^{\mathrm{k}}, 594$ & 1091,8 & 4, ๖3 & $\begin{array}{l}\text { Grappes très } \\
\text { denses Grains } \\
\text { petits, peu mûrs. }\end{array}$ \\
\hline 2 & $\begin{array}{l}\text { Rognage continu. } \\
\text { Effeuillé........ }\end{array}$ & $0^{\mathrm{k}}, 488$ & 1102,3 & 4,20 & $\begin{array}{l}\text { Grappes lon- } \\
\text { gues très denses. } \\
\text { Grains petits, } \\
\text { serrés. }\end{array}$ \\
\hline 3 & Rogné à floraison. & $0^{\mathrm{k}}, 3 \breve{6} 6$ & 1107,8 & 3,83 & $\begin{array}{l}\text { Grappes assez } \\
\text { longues, assez } \\
\text { denses. Grains } \\
\text { gros. }\end{array}$ \\
\hline 4 & Rogné à véraison. & $0^{\mathrm{k}}, 300$ & 1118,0 & 4,23 & $\begin{array}{l}\text { Grappes peti- } \\
\text { tes. assez denses. } \\
\text { Grains gros, non } \\
\text { ratatinés. }\end{array}$ \\
\hline$\check{\jmath}$ & Témoin palissé. . & $0^{\mathrm{k}}, 306$ & 1117,8 & 4,06 & $\begin{array}{l}\text { Grappes peti- } \\
\text { tes, courtes, den- } \\
\text { ses.Grains petits. }\end{array}$ \\
\hline 6 & Témoin. & $0^{\mathrm{k}}, 338$ & 1119,3 & 4,15 & $\begin{array}{l}\text { Grappes lâ- } \\
\text { ches, petites, } \\
\text { quelquefois lon- } \\
\text { gues. Grains } \\
\text { gros. }\end{array}$ \\
\hline
\end{tabular}

Augmentation de production assez importante par le rognage hatif, mais diminution de la qualité. L'effeuillage parait agir favorablement mème sur la qualité des vignes rognées. 


\section{Expérience X. Pinot noir. B. P. 1911}

\begin{tabular}{|c|c|c|c|c|c|}
\hline $\begin{array}{l}\text { Numéros } \\
\text { des } \\
\text { parcelles } \\
\text { - }\end{array}$ & $\begin{array}{c}\text { Nature et époque } \\
\text { du } \\
\text { rognage }\end{array}$ & $\begin{array}{l}\text { Production } \\
\text { par } \\
\text { souche } \\
\text { _ }\end{array}$ & Densité & $\begin{array}{c}\text { Acidité } \\
\text { en S0'Hz2 } \\
-\end{array}$ & Observations \\
\hline 1 & $\begin{array}{l}\text { Rognage continu. } \\
\text { Non effeuillé... }\end{array}$ & $0^{\mathrm{k}}, \breve{570}$ & 1097,8 & 4,16 & $\begin{array}{l}\text { Grappes très } \\
\text { denses, longues. } \\
\text { Grains petits,peu } \\
\text { mûrs. }\end{array}$ \\
\hline 2 & $\begin{array}{l}\text { Rognage continu. } \\
\text { Effeuillé........ }\end{array}$ & $0^{\mathrm{k}}$, วั89 & 1100,8 & 4,08 & $\begin{array}{l}\text { Grappes lon- } \\
\text { gues, den ses. } \\
\text { Grainspeu mûrs. }\end{array}$ \\
\hline 3 & Rogné à floraison. & $0^{\mathrm{k}}, \breve{2} 86$ & 1103,6 & 3,92 & $\begin{array}{r}\text { Grappes den- } \\
\text { ses.Grains petits. }\end{array}$ \\
\hline 4 & Rogné à véraison. & $0^{\mathrm{k}}, 43 \breve{~}$ & 1099,0 & 4,13 & $\begin{array}{l}\text { Grappes peti- } \\
\text { tes, assez denses. } \\
\text { Grains gros, non } \\
\text { ratatinés. }\end{array}$ \\
\hline$\check{\check{z}}$ & Témoin palissé.. & $0^{\mathrm{k}}, 313$ & 1118,8 & 4,05 & $\begin{array}{l}\text { Grappes peti- } \\
\text { tes, courtes, den- } \\
\text { ses. Grains assez } \\
\text { gros. }\end{array}$ \\
\hline 6 & Témoin........ & $0^{\mathrm{k}}, 364$ & 1096,3 & 4,23 & $\begin{array}{l}\text { Grappes lon- } \\
\text { gues, lâches. } \\
\text { Grains gros. }\end{array}$ \\
\hline
\end{tabular}

Influence marquée sur la production, moins nette sur la qualité.

\section{Expérience XI. - Pinot noir. M. 1911}

\begin{tabular}{|c|c|c|c|c|}
\hline $\begin{array}{l}\text { Numéros } \\
\text { des } \\
\text { parcelles } \\
\text { - }\end{array}$ & $\begin{array}{c}\text { Nature } \\
\text { du } \\
\text { rognage }\end{array}$ & $\begin{array}{c}\text { Production } \\
\text { par } \\
\text { souche } \\
\text { - }\end{array}$ & Densité & $\begin{array}{c}\text { Acidité } \\
\text { en SO'H² }\end{array}$ \\
\hline 1 & Rogné. & $0^{\mathrm{k}}, 440$ & 1100,0 & 3,89 \\
\hline 2 & Témoin. & $0^{\mathrm{k}}, 240$ & 1100,0 & 4,17 \\
\hline
\end{tabular}

Forte augmentation de la production ; qualité inchangée. La vigne d'expériences est très touffue.

Expérience XII. - Pinot noir. H. R. 1911

\begin{tabular}{|c|c|c|c|c|}
\hline $\begin{array}{l}\text { Numéros } \\
\text { des } \\
\text { parcelles } \\
-\end{array}$ & $\begin{array}{c}\text { Nature } \\
d u \\
\text { rognage }\end{array}$ & $\begin{array}{c}\text { Production } \\
\text { par } \\
\text { souche } \\
\text { - }\end{array}$ & Densité & $\begin{array}{l}\text { Acidité } \\
\text { en SO }{ }^{\prime} \mathrm{H}^{2}\end{array}$ \\
\hline 1 & Rogné et effeuillé...... & $1^{\mathrm{k}}, 250$ & 1109,0 & 3,95 \\
\hline 2 & Rogné... . . . . . . . . . & $1^{\mathrm{k}}, 272$ & 1102 & 4,10 \\
\hline 3 & Témoin & $1^{\mathrm{k}}, 070$ & 1101 & 4,20 \\
\hline
\end{tabular}


L'augmentation de production est, ici encore, très forte. La qualité n'est pas sensiblement modifiée. La vigne d'expériences est constituée par des souches très touffues.

Le Cabernet-Sauvignon est aussi soumis au rognage dans la plupart des régions où il est cultivé. Malheureusement, je n'ai pu disposer de parcelles en nombre suffisant et assez étendues. Aussi les expériences suivantes ne sont-elles pas aussi probantes que les précédentes. Je les indique néanmoins :

Expérience XIII. - Cabernet-Sauvignon. B. 1909

\begin{tabular}{|c|c|c|c|c|}
\hline $\begin{array}{c}\text { Numéros } \\
\text { des } \\
\text { parcelles } \\
\text { _ }\end{array}$ & $\begin{array}{c}\text { Nature } \\
\text { du } \\
\text { rognage }\end{array}$ & $\begin{array}{l}\text { Production } \\
\text { par } \\
\text { souche }\end{array}$ & Densité & $\begin{array}{l}\text { Aciclité } \\
\text { en SO'H }{ }^{2}\end{array}$ \\
\hline 1 & Rogné à véraison. & $1^{\mathrm{k}}, 692$ & 1083,7 & $\breve{3}, 72$ \\
\hline 2 & Témoin......... & $1^{\mathrm{k}}, 759$ & 1086,7 & $\breve{3}, 83$ \\
\hline
\end{tabular}

Augmentation de production et diminution de la qualité, mais écarts peu importants.

Expérience XIV. - Cabernet-Sauvignon. B. 1910

\begin{tabular}{|c|c|c|c|}
\hline $\begin{array}{l}\text { Numéros } \\
\text { des } \\
\text { parcelles }\end{array}$ & $\begin{array}{c}\text { Nature et époque } \\
\text { du } \\
\text { rognage }\end{array}$ & Densité & $\begin{array}{l}\text { Acidité } \\
\text { en } \\
\mathrm{SO}^{1} \mathrm{H}^{t} \text {. }\end{array}$ \\
\hline- & - & $\stackrel{-}{-}$ & - \\
\hline 1 & Rognage à la véraison & $1086, \breve{3}$ & $\breve{5}, 32$ \\
\hline 2 & Témoin ........... & 1093, 气 & 4,96 \\
\hline
\end{tabular}

Expérience XV. - Cabernet-Sauvignon. M. 1910

\begin{tabular}{|c|c|c|c|c|}
\hline $\begin{array}{l}\text { Numéros } \\
\text { des } \\
\text { parcelles } \\
-\end{array}$ & $\begin{array}{c}\text { Nature et époque } \\
\text { du } \\
\text { rognage }\end{array}$ & $\begin{array}{l}\text { Production } \\
\text { par } \\
\text { souche } \\
-\end{array}$ & Densité & $\begin{array}{c}\text { Acidité } \\
\text { en } \\
\mathrm{SO}^{4} \mathrm{H}^{2} \\
-\end{array}$ \\
\hline 1 & $\begin{array}{l}\text { Rognages successifs à } \\
\text { partir de la floraison }\end{array}$ & $0^{\mathrm{k}}, \breve{\mathrm{o} 00}$ & 1093, こ้ & $\breve{5}, 13$ \\
\hline 2 & A la floraison........ & $0^{\mathrm{k},}, 330$ & $1096, \check{5}$ & 4,95 \\
\hline 3 & Témoin ...... & 0 k, כ้32 & 1097,כٌ & $\breve{\partial}, \breve{\partial}$ \\
\hline
\end{tabular}

Les expériences xirI et xiv ont porté sur des vignes conduites à la méthode du Blayais, où l'on ne rogne guère que lorsque la 
végétation est très touffue. L'expérience xv sur des vignes conduites d'après la méthode du Médoc, où l'on rogne plusieurs fois après la floraison. Dans tous les cas, la qualité a été diminuée.

Expérience XVI. - Cabernet-Sauvignon. B. 1910.

\begin{tabular}{|c|c|c|c|c|}
\hline $\begin{array}{c}\text { Numéros } \\
\text { des } \\
\text { parcelles }\end{array}$ & $\begin{array}{c}\text { Nature } \\
\text { du } \\
\text { rognage }\end{array}$ & $\begin{array}{l}\text { Production } \\
\text { par } \\
\text { souche }\end{array}$ & Densité & $\begin{array}{c}\text { Acidité } \\
\text { en } \\
\mathrm{SO}^{4} \mathrm{H}^{2}\end{array}$ \\
\hline 1 & Témoin palissé & $1^{\mathrm{k}}, 221$ & 1094 & $3, \breve{0}$ \\
\hline 2 & Rogné ... . . . . & $0^{\mathrm{k}}, 978$ & 1083 & 4,18 \\
\hline
\end{tabular}

Les rameaux de 1 ont été maintenus verticaux sur toute leur longueur. L'écart est donc ici, la résultante et du palissage vertical et du rognage. La part de ce dernier ne peut pas ètre mise en évidence.

Expérience XVII. - Cabernet-Sauvignon. M. 1911.

\begin{tabular}{|c|c|c|c|c|}
\hline $\begin{array}{l}\text { Numéros } \\
\text { des } \\
\text { parcelles } \\
-\end{array}$ & $\begin{array}{c}\text { Nature } \\
\text { du } \\
\text { rognage }\end{array}$ & $\begin{array}{l}\text { Production } \\
\text { par } \\
\text { souche } \\
\text { - }\end{array}$ & Densité & $\begin{array}{l}\text { Acidité } \\
\text { en } \\
\mathrm{SO}^{4} \mathrm{H}^{2} \\
-\end{array}$ \\
\hline 1 & Rogné plusieurs fois. & $0^{\mathrm{k}}, 787$ & 1086 & 4,07 \\
\hline 2 & Rogné 1 fois....... & $0^{\mathrm{k}}, \breve{118}$ & 1096 & 3,40 \\
\hline 3 & Palissé... & $0^{\mathrm{k}}, 431$ & 1097 & 3,41 \\
\hline
\end{tabular}

Mèmes résultats qu'en 1910; augmentation très forte de la production sur les rangs rognés plusieurs fois.

\section{ROGNAGE DES ENTRE-COEURS}

Les rognages hâtifs font développer, surtout sur les vignes vigoureuses, un nombre souvent considérable de ramifications secondaires. Ces jeunes rameaux peuvent ètre laissés intacts ou réduits à une ou deux feuilles. Leur réduction fait apparaitre des ramifications tertiaires qui sont réduites, à leur tour, à úne ou deux feuilles et ainsi de suite.

Ces rognages successifs sont l'équivalent dı rognage dles 
rameaux primaires. Ils doivent avoir les mèmes eonséquences et les tableaux précédents montrent, en effet, qu'ils accentuent souvent la diminution de la qualité, retardent l'aoùtement, etc.

\section{SUPPRESSION DES ENTRE-COEURS}

Au lieu de réduire les ramifications à une ou deux feuilles, on les supprime quelquefois en totalité (Suisse). Le rameau ne porte alors que les $8,10,12$ feuilles de sa partie inférieure. La surface foliaire très réduite, est ainsi maintenue constante. Qu'en résulte-t-il?

Expérience XVIII. - Folle Blanche. 1896.

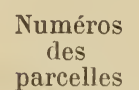

parcelles

$\overline{1}$

2

3

3 Rognée et entre-cœurs

$$
\begin{gathered}
\text { Nature } \\
\text { du } \\
\text { rognage }
\end{gathered}
$$

Rognnée et entre-cœurs

supprimés .......

Témoin............

Rognee et entre-cœurs

Témqin .............
Densité

1037

1069

1052

$105 \%$
Acidité

8,22

10,00

L'expérience xvıı montre que les raisins des vignes rognées n’ont pu arriver à maturité. Les feuiles primaires sont devenues très épaisses et les sarments n'ont pu s'aoùter. Ainsi, une vigne de Folle Blanche très fertille sous le climat du sud-ouest ne peut amener ses raisins à bonne maturité quand elle est nourrie par un nombre constant et limité de feuilles adultes.

Dans d'autres expériences faites sur l'Aramon, au lieu de rogner les sarments primaires, on les a laissés s'allonger librement et, comme dans le cas précédent, on a supprimé les ramifications dès qu'elles se montraient.

La vigne est nourrie exclusivement par ses feuilles primaires dont le nombre est variable et il en résulte ce qui suit: 
Expérience XIX. - Aramon. 1905.

Numéros
des

parcelles

$\overline{1}$

2 $\begin{gathered}\text { Désignation } \\ \text { de }\end{gathered}$
l'expérience

Souche sans entre-cœurs

Témoin .............
Densité

$106 \overline{3}, 8 \quad 5, \overline{30}$

$1069,3 \quad 4,90$

Expérience XX. - Aramon. 190\%.

\begin{tabular}{|c|c|c|c|c|c|}
\hline $\begin{array}{l}\text { Numéros } \\
\text { des } \\
\text { parcelles }\end{array}$ & $\begin{array}{c}\text { Désignation } \\
\text { de } \\
\text { l'expérience }\end{array}$ & $\begin{array}{l}\text { Poids de } \\
\text { récolte } \\
\text { par souche }\end{array}$ & $\frac{\mathrm{F}}{\mathrm{v}}$ & Densité & Acidite \\
\hline$\overline{1}$ & Sans entre-cœurs & & 5,66 & 1060,9 & \\
\hline 2 & Témoin........ & & 5,57 & 1058,9 & \\
\hline \multicolumn{6}{|c|}{1908} \\
\hline 1 & Sans entre-cœurs & & 10,32 & $106 \Xi, 8$ & $\breve{5}, 30$ \\
\hline 2 & Témoin ... . . . . . & & 7,56 & 1069,3 & 4,90 \\
\hline \multicolumn{6}{|c|}{1909} \\
\hline 1 & Sans entre-cœurs & $4^{k}, 38$ & 6,92 & 1053,9 & 6,34 \\
\hline 2 & Témoin ........ & $\breve{3}^{\mathrm{k}}, 06$ & 6,33 & $103 ̋ 4,7$ & $\check{5}, \check{59}$ \\
\hline \multicolumn{6}{|c|}{1910} \\
\hline 1 & Sans entre-cœurs & $3^{\mathrm{k}}, 73$ & 6,00 & 1059,8 & 6,22 \\
\hline 2 & Témoin ........ & $\breve{5}^{\mathrm{k}}, 07$ & 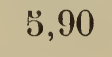 & 1060,8 & 7,62 \\
\hline \multicolumn{6}{|c|}{1911} \\
\hline 1 & Sans entre-cœurs & $2^{\mathrm{k}}, 113$ & & 1067 & 3,75 \\
\hline 2 & Témoin........ & $2^{\mathrm{k}}, 700$ & & 1068 & 3,89 \\
\hline
\end{tabular}

Il y a régulièrement diminution de la qualité, mais l'écart est bien faible. En serait-il de même ailleurs et chez d'autres cépages? En tout cas, cette expérience montre que le feuillage des ramifications secondaires n'est pas toujours absolument indispensable à la maturation des fruits ; les feuilles primaires peuvent presque y suffire. Au reste, les rameaux rectilignes, dont les rameaux secondaires sont peu développés, sont aussi ceux qui assurent aux grappes le maximum de qualité. La ramification apparaît, dès lors, comme un cas anormal : le résultat d'un accident, courbure, blessure, sections partielles ou totales, impuissance du bourgeon terminal à s'accroittre.... 
INFLUENCE DU ROGNAGE SUR LE GROSSISSEMENT DE LA GRAPPE ET DES GRAINS

L'augmentation de récolte consécutive aux rognages hàtifs n'est pas due, sauf peut-ètre dans des cas très rares, que nous n'avons pas, du r'este, rencontrés, à l'augmentation du nombre

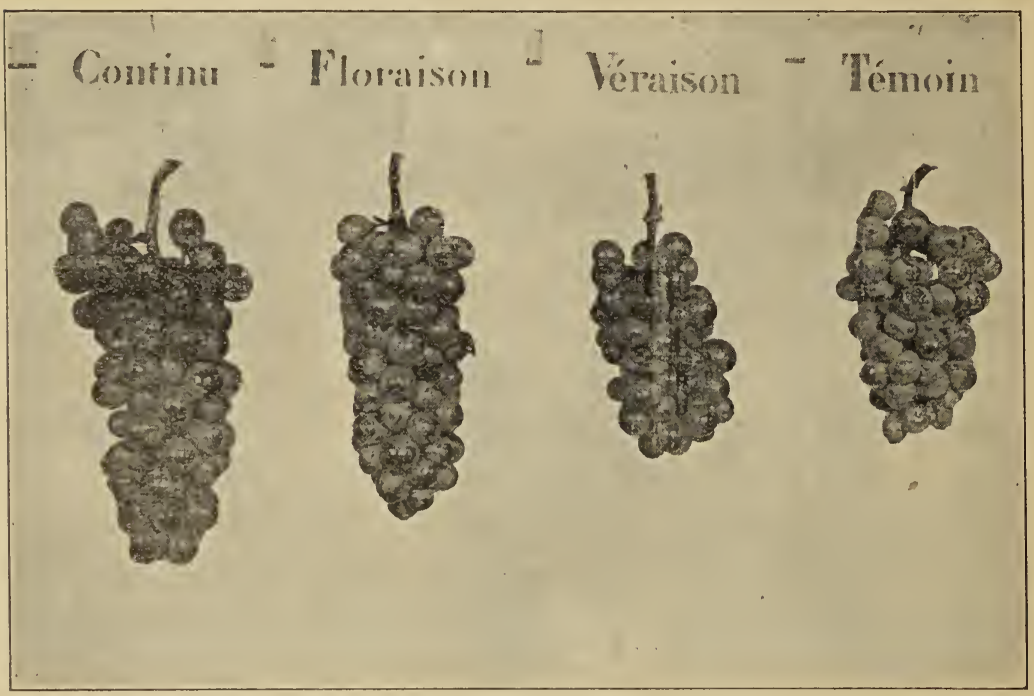

Fig. 1. - Grappes de Pinot.

de grappes. A l'époque où il est pratiqué, le rognage n'en peut faire apparaitre de nouvelles sur les rameaux primaires. Elle est donc due à l'accroissement du poids de chacune d'elles. (Fig. 1 et 2).

C'est ce qui est bien mis en évidence dans le tableau suivant:

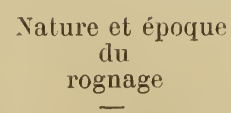

Rognage à la floraison

Rognage continu.....

Témoin ...........

$\frac{\text { Poids moyen des grappes de Pinol }}{1909} 1910 \quad 1911$

$\begin{array}{lll}0^{\mathrm{k}}, \overline{111} & 0^{\mathrm{k}}, \overline{103} & 0^{\mathrm{k}}, \mathbf{1 1 8} \\ & 0^{\mathrm{k}}, 103 & 0^{\mathrm{k}}, 132 \\ 0^{\mathrm{k}}, 101 & 0^{\mathrm{k}}, 077 & 0^{\mathrm{k}}, 063\end{array}$


Les différences sont, ici, extrêmement nettes. Sont-elles la conséquence du grossissement des grains ou de l'augmentation du nombre de ces derniers?

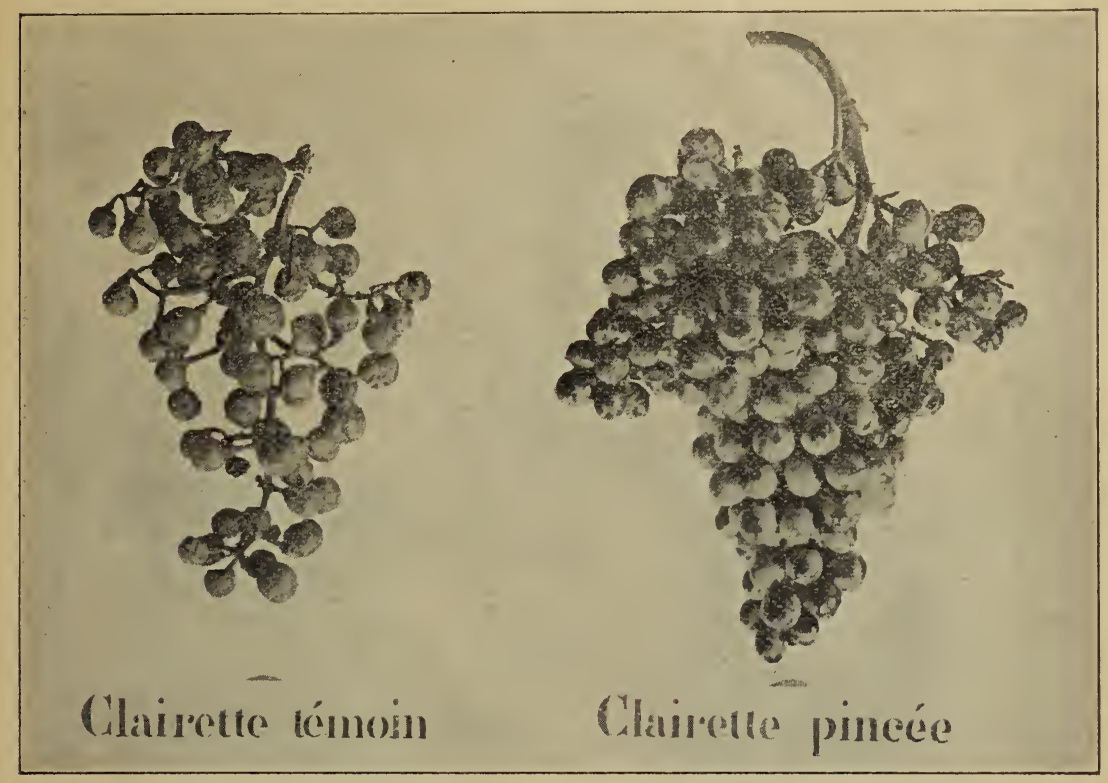

Fig. 2. - Grappes de Clairelle.

Le grossissement des grains, qu'Olivier de Serres a déjà constaté, a bien été mis en évidence par les recherches de M. Duclou. Voici quelques-uns de ses résultats :

\begin{tabular}{|c|c|c|c|c|c|c|}
\hline & \multicolumn{2}{|c|}{ MALBEG } & \multicolumn{2}{|c|}{ MERLOT } & \multicolumn{2}{|c|}{$\mathrm{C}_{\text {ABERNET-S}}$ AUVIGNON } \\
\hline & Témoin & Rogné & Témoin & Rogné & Témoin & Rogné \\
\hline Jus. & $75,70^{\circ} \%$ & $77 \%$ & $72,80 \%$ & $76,20^{\circ} \%$ & $70,80^{\circ} \%$ & $71 \%$ \\
\hline Pédoncules & $5,50 \%$ & $5,10^{\circ} / \mathrm{s}$ & $6,70 \%$ & $5,20^{\circ} / \circ$ & $5,10 \%$ & $5,20 \%$ \\
\hline
\end{tabular}

Savastano, Pacottet et Jeanin, n'ont pas observé une pareille influence ; ils ont mème constaté le. plus souvent une réduction du volume.

En 1911, j'ai fait déterminer le volume des grains mùrs de Pinots soumis à diverses pratiques culturales. Voici les résultats obtenus : 
Expérience XXI. - Volume des grains de Pinot noir. 1911.

\begin{tabular}{|c|c|c|}
\hline $\begin{array}{l}\text { Numéros } \\
\text { des } \\
\text { parcelles }\end{array}$ & $\begin{array}{c}\text { Nature et époque } \\
\text { du } \\
\text { rognage }\end{array}$ & $\begin{array}{c}\text { Volume d'un grain } \\
\text { en } \\
\text { centimètres cubes }\end{array}$ \\
\hline 1 & Rognage continu. . & $\overline{1}$ \\
\hline 2 & $-\quad \ldots \ldots \ldots$ & 1 \\
\hline 3 & et effeuillage. & 1.28 \\
\hline 4 & et effeuillage. & 1.138 \\
\hline$\check{3}$ & Rognage à floraison......... & 1.214 \\
\hline 6 & $-\quad \ldots \ldots \ldots$ & 1.07 \\
\hline 7 & Rognage à véraison..... & 1.323 \\
\hline 8 & $-\quad \ldots$ & 0.980 \\
\hline 9 & Témoin.. . & 1.28 \\
\hline 10 & Témoin.... . & 0.742 \\
\hline 11 & Témoin.................. & 0.789 \\
\hline
\end{tabular}

L'augmentation du volume des grains n'est pas constante : marquée et mème importante pour un des rognages pratiqués à la véraison, elle est très faible ou nulle et mème négative pour d'autres. Pourquoi ? D'abord, le grossissement des grains est fonction non seulement de cette opération, mais encore deleur nombre. On conçoit donc que les grains des vignes rognées puissent ètre plus petits que ceux des témoins. Mais ils sont aussi quelquefois plus gros. C'est que l'accroissement de leur nombre peut ètre assez faible pour ne pas compenser les effets directs du rognage sur la croissance.

Mais comment expliquer la diminution du poids des grappes signalées par quelques observateurs, c'est-à-dire une réduction de la récolte ? Peut-être de la manière suivante : si le rognage met d'abord à la disposition des grappes des substances en quantité considérable, il réduit la surface foliacée et, par conséquent, l'assimilation. La réduction peut être quelconque. Très petite, elle n'a pas d'influence appréciable sur la nutrition des grappes : plus grande, comme quand le rognage amène la suppression de la moitié du feuillage, elle entraine une alimentation insuffisante des grappes. 
On admet que les rognages ont aussi pour objet d'arrêter la végétation et, en conséquence, de hâter la maturité des fruits des bois et des feuilles.

"Lorsque le raisin va mùrir, dit M. Mazade, il faut que le développement de la partie végétative soit arrèté au bénéfice de la maturation ».

On a vu ce qu'il en était dans la pratique. Quant aux sarments, «il en favorise, suivant Cazaux-Cazalet, le grossissement et l'aoùtement. Le meilleur aoùtement des vignes rognées a été

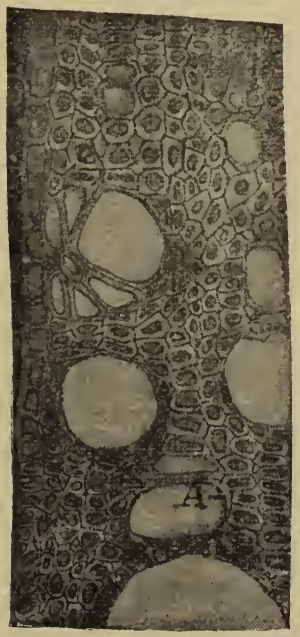

Fig. 3. - Le bois dans un sarment rogné ; en A, les fibres ligneuses = redeviennent plus épaisses radialement.

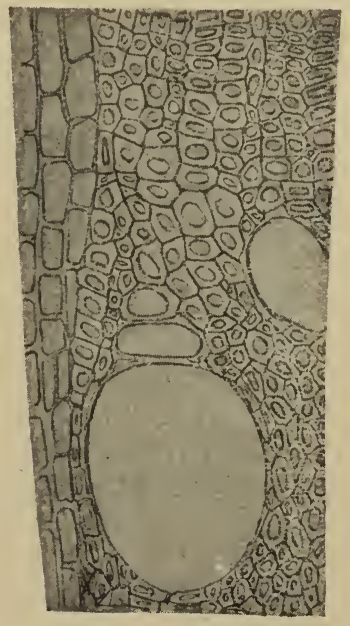

Fig. 4. - Le bois dans un sarment dressé et non rogné.

constaté pendant plusieurs.années au microscope, en recherchant l'état de la lignification et l'amidon : on l'a reconnu aussi à la dureté des bois rognés ».

Une coupe microscopique, à travers des régions de mème àge, montre les différences de constitution tissulaire qui existent entre les sarments rognés et les témoins. 
L'épaisseur radiale des cellules du bois, qui est d'abord allée en diminuant lentement de l'intérieur à l'extérieur, s'accroît à nouveau tout de suite après le rognage, fig. $3 \mathrm{~A}$ et fig. 4. Mais à l'automne, ces nouvelles régions paraissent moins riches en amidon que les anciennes ; les vaisseaux y sont plus nombreux et cela se conçoit. Le parenchyme ligneux est peu lignifié, de

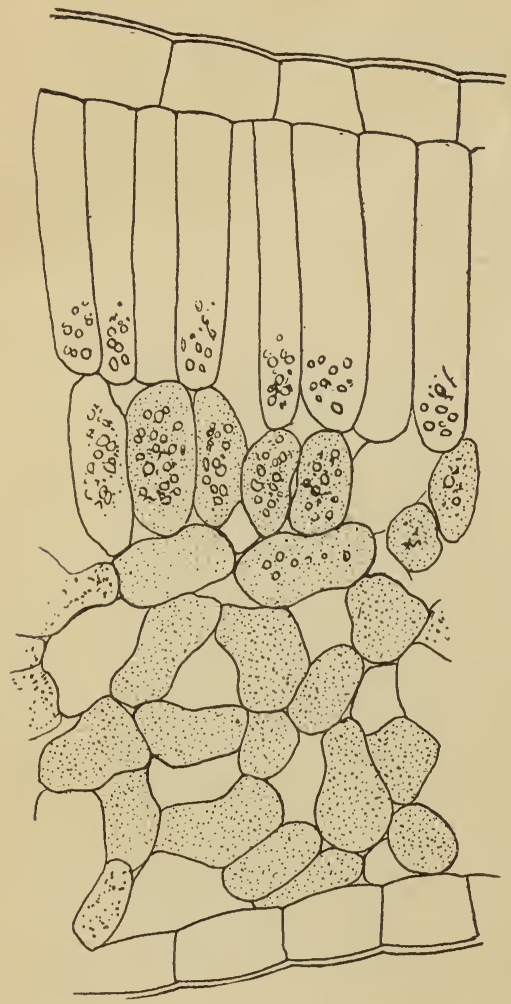

Fig. 5. - Coupe transversale d'une feuille de rameau rogné; elle a deux assises de cellules en palissade.

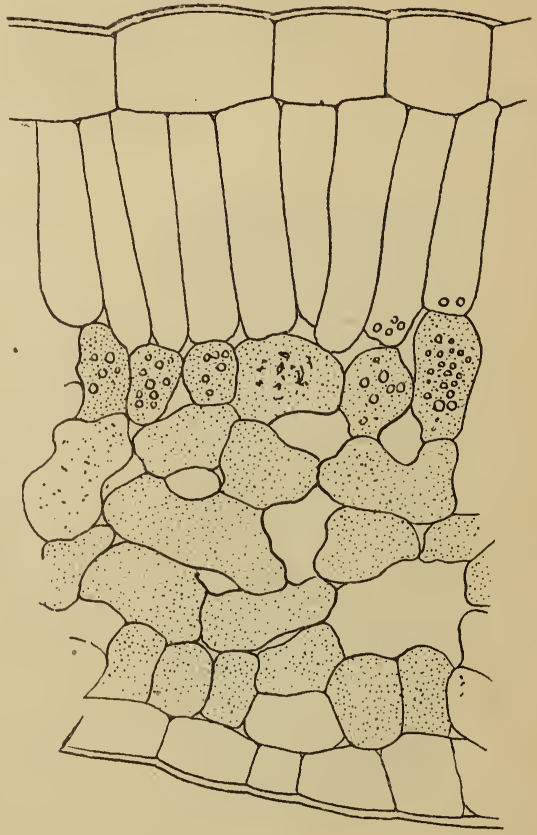

Fig. 6. - Coupe transversale d'une feuille de rameau dressé et non rogné.

mème que la couronne. Ces modifications de la structure existent sur toute la longueur de la partie conservée, mais elles sont d'autant plus accusées que les tissus sont plus voisins de la section terminale. Existent-elles aussi sur les racines? On les retrouve pas nettement accusées sur les racines d'un an, dont l'épaississement est cependant rapide ; elles sont encore moins 
faciles à constater sur les couches minces de croissance du tronc et des bras.

Le rognage fait donc grossir les sarments; cela n'est pas douteux. Et peut-ètre fait-il aussi grossir et allonger les racines.

Et l'on voit qu'au lieu d'arrèter la végétation, comme on le croit, il la prolonge; qu'au lieu de hàter l'aoùtement, il le retarde, il augmente l'activité des tissus qui peuvent s'accroître ; il ne la réduit point. Et voilà encore une raison qui explique la diminution de la qualité chez les vignes rognées.

Il en est si bien ainsi, que ces vignes, au lieu de prendre hâtivement la teinte d'automne, conservent leur feuillage vert fort longtemps; d'autant plus, du reste, que les rognages ont été plus nombreux. Elles se colorent aussi vivement en rouge, alors que les témoins prennent une teinte jaunàtre de plus en plus pâle.

Les feuilles s'accroissent aussi nettement en épaisseur, fig. こ̆ et 6 ; on le constate facilement à la main.

La prolongation de la végétation peut être obtenue par des fumiers, des arrosages qui aboutissent aussi à un accroissement du volume des grains. Le rognage, à cè point de vue, est leur équivalent et il explique l'accroisssement du volume des grains signalé par Duclou, etc.

INFLUENCE DU ROGNAGE SUR LA PUISSANCE DE LA VIGNE

La plupart de nos expériences sur Pinot, Cabernet, etc., ont porté, chaque année, sur des parcelles différentes. On n'a pu, par suite, connaìtre le retentissement de cette opération sur la végétation des années suivantes. Et, d'autre part, si le poids des sarments donne annuellement la puissance des vignes normales, il est nécessairement réduit pour les vignes rognées.

Seule, l'allure de la courbe de la puissance annuelle portant sur plusieurs années, donne des indications utiles. Les aramons de Claparède ont donné les poids suivantes : 
Poids de la végétation par souche

\begin{tabular}{|c|c|c|c|c|c|c|}
\hline $\begin{array}{l}\text { Numéros } \\
\text { des } \\
\text { parcelles }\end{array}$ & $\begin{array}{c}\text { Nature } \\
\text { du } \\
\text { rognage }\end{array}$ & 1906 & 1907 & 1908 & 1909 & 1910 \\
\hline- & - & - & - & - & - & - \\
\hline 1 & Très hàtif. . & 0,470 & 0,6 8 & $0,470^{1)}$ & $0,67 \check{~}$ & 0,718 \\
\hline 2 & A floraison & 0,320 & 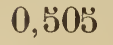 & 0,470 & $0,64 气$ & 0,764 \\
\hline 3 & $\begin{array}{l}\text { Après florai- } \\
\text { son ...... }\end{array}$ & $0, \check{5} 40$ & 0,347 & 0,400 & 0,641 & $0,91 \check{ }$ \\
\hline 4 & A véraison.. & $0, \check{2} 20$ & 0,767 & 0,6 əั8 & 0,811 & $0,83 \check{0}$ \\
\hline วั & Témoin..... & $0, \check{2} 20$ & 0,788 & 0,617 & $0,803 ٌ$ & 0,847 \\
\hline
\end{tabular}

Dans le Midi de la France, on admet volontiers que le rognage affaiblit la souche. Quand il en est ainsi, on peut se demander si l'augmentation de la production n'est pas la cause réelle de l'affaiblissement. Dans certains cas, son ròle est sùrementprédominant. Mais si la production n'est pas modifiée, pratiqué une seule fois, il doit avoir bien peu d'influence ; plusieurs fois, il en est nécessairement autrement. (Voir plus loin).

L'enlèvement des entré-cœurs donne à la puissance de la végétation l'allure indiquée dans le tableau ci-après :

\section{TABLEAU}

\begin{tabular}{|c|c|c|c|c|c|c|}
\hline \multirow{3}{*}{$\begin{array}{c}\text { Numéros } \\
\text { des } \\
\text { parcelles } \\
\text { - }\end{array}$} & \multirow{3}{*}{$\begin{array}{c}\text { Nalure } \\
\text { du } \\
\text { rognage } \\
\end{array}$} & \multicolumn{5}{|c|}{ Poids des sarments par souche } \\
\hline & & 1906 & 1907 & 1908 & 1909 & 1910 \\
\hline & & - & - & - & - & - \\
\hline 1 & Sans entre- & kil. & kil. & kil. & kil. & kil. \\
\hline & cneurs. & 0,306 & 0,707 & 0,413 & 0,626 & 0,640 \\
\hline 2 & Témoin. . . & 0,466 & 0,647 & 0,446 & 0,800 & $0,8 \breve{3}$ \\
\hline
\end{tabular}

Il semble bien que l'écart des poids de la végétation tende à s'accroìtre. L'enlèvement continu des entre-cœurs serait ainsi, à la longue, une cause d'affaiblissement de la vigne. - Mais il s'agit ici de vignes non palissées. 
Dıscussion. - Des expériences qui précèdent, il résulte que le rognage :

A. Modifie la production : $1^{\circ}$ Il l'augmente en augmentant : $a$ ) le nombre ; $b$ ) le volume des grains.

$2^{\circ}$ Il la diminue en réduisant le volume des grains.

B. Il modifie la qualité du raisin et du vin. 10 Il la diminue généralement en retardant la maturité.

$2^{\circ}$ Il l'améliore aussi.

C. Quand les effets de cette pratique paraissent ètre nuls, c'est qu'en réalité, ils ont été trop peu marqués, dans un sens ou dans l'autre, pour ètre mesurés par les moyens dont nous avons fait usage.

D. Il modifie l'activité ou la durée de la végétation. $1^{\circ}$ Il l'accroit généralement.

Tous ces effets sont hors de doute. Bien que contradictoires, tous doivent ètre retenus. Il nous reste à lever la contradiction en les éludiant maintenant d'un peu plus près.

A. Modification de la production. $1^{\circ}$ Augmentation du nombre de grains. - Elle n'est obtenue que par les rognages hàtifs, - c'est-à-dire pratiqués avant, pendant et un peu après la floraison - qui favorisent le développement des jeunes fleurs et grappes et empêchent aussi l'avortement ou le filage de ces dernières, et s'opposent à la chute des fleurs ou des jeunes grains. Dans les deux cas, ils évitent la coulure. Comment? Cette question a fait l'objet de plusieurs travaux remarquables de Müller-Thurgau, Cuboni, etc.

Müller-Thurgau en établissant le ròle des feuilles de la vigne dans la production du sucre et l'élaboration des matières plastiques a, un des premiers, montré les conséquences du rognage sur la vigne.

« En respirant, les cellules de la vigne consomment du sucre et l'acide carbonique résultant de cette combustion, est expiré en majeure partie. Si maintenant nous réfléchissons que ce n'est pas seulement le protoplasma des cellules de la feuille qui respire, mais aussi les innombrables corpuscules de protoplasma 
de toutes les cellules vivantes, par conséquent aussi ceux des bourgeons, des racines, etc., et que ce phénomène dure sans interruption jour et nuit depuis le printemps jusqu'à l'automne, nous nous expliquerons qu'une vigne consomme en respirant des quantités fort considérables de sucre.

Nous'savons, en outre, que la cellulose dont se constituent les innombrables parois des cellules, est également formée de sucre et que les substances albuminoïdes du protoplasma proviennent de sucre et d'un sel d'azote. Alors, quand il se forme de nouvelles pousses ou des fenilles nouvelles ou bien que les racines s'allongent et que le tronc gagne en épaisseur, la vigne a besoin pour tout cela, de quantités considérables de sucre et il se trouve que le sucre emmagasinédans les raisins, n'est qu'une petite partie de la quantité de sucre employé par la vigne ; la plus grande partie est consommée par la respiration et la croissance. »

De telle sorte qu'en supprimant les organes en voie de croissance - et ce sont les extrémités des rameaux - on rend disponible, pour les grappes, une plus grande quantité de matières sucrées.

Dans un travail important (1), Guboni a précisé le ròle des feuilles d'àge différent dans la formation de l'amidon. D'après ses expériences, pendant le premier mois de la végétation, les jeunesfeuilles ne produisent pas d'amidon. Celui-ci ne commence à apparaitre que sur les feuilles basilaires des rameaux ayant environ 30 centimètres ; en conséquence, les jeunes rameaux vivent en parasites aux dépens des matériaux nutritifs emmagasinés dans la plante durant de l'année précédente.

Et cette incapacité des jeunes feuilles à produire de l'amidon, on la retrouve, durant l'été, chez toutes les feuilles de formation récente.

Une fois devenues adultes ou presque adultes, les feuilles

1) Richerche sulla formazione dell amidon nelle foglie della vite1885. 
produisent de l'amidon tant qu'elles sont vertes, mème à l'automne et longtemps après la vendange. Il est facile de s'en assurer par l'épreuve à l'iode. Mais dès que leur couleur se modifie, qu'elles deviennent jaunâtres ou rouges, leur capacité de former de l'amidon s'atténue pour disparaitre complétement chez les feuilles jaunes.

Et de ces résultats, M. Cuboni tire les conclusions suivantes : “ L'opération appelée taille en vert ou rognage, conseillée par quelques-uns et combattue par d'autres, trouve ici une explication scientifique plausible. En effet, il est évident que les entrecœurs et les sommets n'étant pas aptes à élaborer l'amidon, mais en consomment plus tòt au préjuđaice du sucre qui se rendrait dans les grappes, devraient ètre éliminés quand on sait qu ils ne pourront arriver à temps à l'état adulte pour contribuer à la maturation du raisin. »

M. Griffon a repris l'étude des fonctions des jeunes feuilles par la méthode de Boussingault et il constate que les plus jeunes feuilles épanouies, si elles ne forment pas de l'amidon, assimilent l'acide carbonique et que, par suite, «il est exagéré de prétendre que l'opération culturale, dite de l'écimage de la vigne, est justifiée exclusivement par ce fait que les rameaux supprimés vivaient en parasites sur les parties inférieures. Ce parasitisme est très faible ou nul selon l'éclairement; il peut mème ne jamais exister si l'on considère une pousse portant une ou 2 feuilles bien développées. Le reflux de la sève vers les sarments, les fruits et les souches, le bon équilibre entre la partie foliaire restante et la masse des raisins sont bien plutòt, comme on l'a souvent avancé, les vraies causes des bons effets que l'on attribue à l'écimage. »

Que les jeunes feuilles puissent décomposer $\mathrm{CO}^{2}$, cela n'est pas douteux ; elles renferment de la chlorophylle qui peut, par beau temps, décomposer plus d'acide carbonique que la respiration des tissus n'en détruit. Aussi bien la véritable question n'est-elle point là. Il s'agit, en définitive, de savoir si ces feuilles, auxquelles il faut ajouter les mérithalles et les vrilles, assimilent plus de carbone qu'elles n'en brùlent dans 24 heures. Et il semble bien que, pour les jeunes rameaux, la consommation 
dépasse la production ; car au carbone brùlé, il faut ajouter celui qui est utilisé pour la construction des tissus nouveaux, qui est surtout active aux extrémités en voie de croissance (rameaux et racines).

On peut donc conclure que les extrémités en voie de croissance sont des sortes de parasites, quise constituent surtout aux dépens des matières élaborées ou transformées dans les autres parties de la plante. Leur suppression, par les rognages, rend disponibles, pour les grappes, les matières qu'elles auraient utilisées pours'accroitre; et les grains, désormais largement alimentés, grossissent et évoluent normalement. Les rognages sont, par suite, d'autant plus efficaces qu'ils portent sur des rameaux plus vigoureux ou des souches plus puissantes; et c'est, en effet, chez les vignes à croissance rapide: Glairette, Grenache, Pineau, etc., 'ou greffées sur puissant sujet : Rupestris, 1202, etc., qu'il augmentent le plus la production.

Tous les éléments rendus disponibles par la suppression des sommets des rameaux, ont-ils la mème valeur? Müller Thurgau et Cuboni croient surtout au rôle du sucre. Les analyses suivantes de grains de Grenache montrent qu'en définitive, les jeunes grains avortés et tombés à terre, sont plus riches en éléments minéraux que les grains normaux.

\begin{tabular}{|c|c|c|}
\hline & \multicolumn{2}{|c|}{$\begin{array}{l}\text { Composition o/o ramenée à la matière } \\
\text { sèche des grains de raisins }\end{array}$} \\
\hline & coulés & normaux \\
\hline Azote. & 2,87 & $2, \overline{37}$ \\
\hline $\mathrm{K}^{2} \mathrm{O} \ldots$ & 2,08 & 1,76 \\
\hline $\mathrm{P}^{2} 0^{5} \ldots$ & 1,09 & 0,959 \\
\hline M. g. o.... & 0,492 & 0,423 \\
\hline Саo........ & 2,05 & 1,88 \\
\hline Fer............. & 0,015 & 0,006 \\
\hline Matière minérale $o / o \ldots$ & $8, \breve{0}$ & 6,70 \\
\hline
\end{tabular}

L'élément qui manque ici est le carbone. C'est donc lui qui joue probablement le rôle le plus important et, ce qui le montre c'est que le beau temps, qui favorise l'accumulation du sucre 
dans les tissus favorise aussi le développement des fleurs et des grains. La coulure est rare parles journẻes bien ensoleillées.

$2^{\circ}$ Augmentation du volume des grains. - Les rognages tardifs ne peuvent guère modifier le nombre des grains. Mais ils rendent néanmoins disponibles des matières nutritives et notamment des sucres, que les grappes peuvent employer pour activer la croissance et augmenter le volume de leurs grains.

Mais l'augmentation du nombre et l'accroissement du volume des grains sont un seul et mène phénomène : l'accroissement du volume du fruit. Et il s'agit maintenant de rechercher si l'efficacité des rognages reste constante, quelle que soit l'époque à laquelle on les pratique.

Si l'on compare dans les tableaux précédents, les poids des grappes des vignes rognées hàtivement au poids des grappes des vignes rognées tardivement, il apparait, tout de suite, un écart très grand : les rognages tardifssont peu efficaces. A quoi cela tient-il ? Simplement, sans doute, à ce que l'importance des extrémités parasites diminue sans cesse, non pas seulement en valeur absolue, mais surtout en valeur relative (fig. 7). En valeur absolue, la surface parasite peut ètre re-

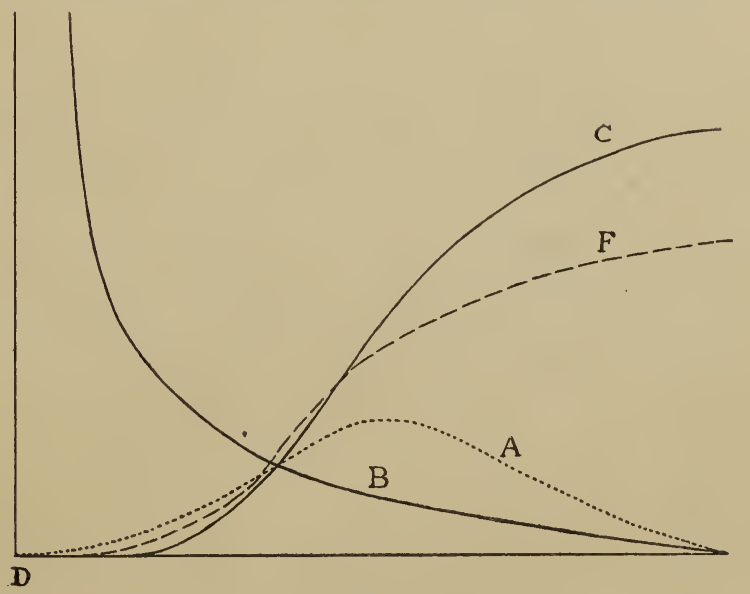

Fig. 7.

présentée par la courbe A. Elle est d'abord très faible, devient . maxima sensiblement à l'époque de la floraison, puis décroit et 
devient nulle un peu après l'arrèt de la végétation. S'il y a une pousse d'aoùt, elle reprend des valeurs lentement croissantes.

La courbe $\mathrm{G}$ représente la surface non parasite des mèmes rameaux ; elle croìt sans cesse jusqu'à l'arrèt de la végétation. C'est le rapport de la courbe A à la courbe $\mathrm{C}$, qui donne la mesure de l'importance relative des sommets parasites; il peut ètre exprimé par la courbe $B$ dont les valeurs, d'abord très grandes, s'annulent en mème temps que celles de A. C'est dire que le ròle des extrémités parasites est immense au départ de la végétation ; il est encore important au moment de la floraison et il est nul un peu après l'arrèt de la végétation. Or, le rognage doit réduire la valeur de ce rapport et mème l'amener à zéro. Comme le dénominateur $\mathrm{C}$ est d'abord très faible, la réduction de A doit ètre très forte pour atteindre ce résultat. Mais $\mathrm{G}$ grandit très vite : le rapport $\frac{\mathrm{A}}{\mathrm{C}}$ tend donc par ce seul fait, vers zéro ; et la réduction de $\mathrm{A}$, c'est-à-dire le rognage, devient de moins en moins nécessaire à mesure que les rameaux vieillissent.

Quand A égale zéro, c'est-à-dire quand toutes les extrémités ont cessé de grandir, le rognage est inutile ou même nuisible. Si on le pratique, on supprime des organes utiles.

B. Le rognage modifie la qualité du raisin et du vin. $1^{\circ}$ Il la diminue en retardant la maturité. Et cependant, la suppression des sommets parasites devrait, semble-t-il, puisqu'ils consomment du carbone, aboutir à un résultat contraire. Mais à l'époque de la maturation, de nouveaux sommets parasites ont déjà remplacé ceux que les rognages hâtifs ont supprimé. Quant aux rognages tardifs, même avec une végétation encore active, le rapport $\frac{\mathrm{A}}{\mathrm{B}}$ est déjà si faible qu'ils ne peuvent guère le réduire d'une quantité appréciable.

D'autre part, $1^{\circ}$ la surface foliacée totale reste réduite : première cause de diminution de la qualité.

$2 \circ$ La production est augmentée et toute augmentation de pro-

- duction entraine une réduction de la qualité: 
$3^{\circ}$ La prolongation de l'état de vie active est aussi une eause de réduction de la qualité.

Il améliore la qualité. C'est ce qu'élablissent plusieur's des tableaux précédents, mais l'amélioration n'a été appréciable que pour les vignes touffues, dont les grappes sont insuffisamment éclairées. Le rognage joue ici partiellement, le ròle du palissage, de l'effeuillage (1). Son influence nuisible est toujours réelle, mais elle peut ètre largement compensée par un meilleur éclairement des raisins.

C. Sur l'Aramon, les effets du rognage ont été peu apparents. C'est qu'ils ne sont pas seulement fonction de $\frac{\mathrm{A}}{\mathrm{C}}$, mais encore de la masse de fruits portée parles rameaux, c'est-à-dire de $\frac{\mathrm{F}}{\mathrm{V}}$. Chez les Pinots, $\frac{F}{V}$ est très petit; la suppression des sommets parasites profite à un faible poids de raisins et son action derient ainsi netlement apparente par l'accroissement du nombre ou du volume des grains. Chez l'Aramon, le poids des raisins F (fig. 7) est considérable: les effets de la suppression des sommets sont, parsuite, moins nets dans l'un et l'autre sens.

D'autre part, les dimensions des grains peuvent varier che\%ce entre cépage des limites très étendues et l'accroissement de volume compense la réduction du nombre. Cependant, il y a des cas, et il est facile de les concevoir, où le rognage doit avoir les mèmes effets que sur les Pinots. Le cas de l'Aramon doit ètre celui de tous les cépages grros producteurs.

Et enfiı, les Aramons qui ont servi pour nos expériences, étalent librement leurs rameaux sur le sol : ceux-ci se recourbent nécessairement dès que leur poids devient suffisant; les courbures, lont comme on sait, apparaitre et développer des ramifications secondaires. Or, le rognage fait aussi apparaitre des ramificalions. La courbure est, par suite, presque l'équivalent d'un rognage. Pratiquer ce dernier sur des rameaux recourbés, c'est faire une opération qui est déjà faite.

(1). L. Ravaz. L'effeuillage de la vigne. 
D. Le rognage prolonge l'état de vie active. - Il le prolonge tout comme la courbure des rameaux : en somme, en concentrant l'activité végétative près du corps de la souche. En conséquence, tout ce qui tend à raccourcir les sarments, devrait aboutir au mème résultat. Le court-noué agit bien ainsi. Iu fond, le rognage agit ici comme sur les grappes, enmettant à la disposition des tissus restants, l'eau et les substances qui auraient été utilisées par les sommets. Chez les vignes non palissées (Aramon), l'effet apparent est sensiblement nul pour la raison exposée plus haut.

Peut-il diminuer l'activité de la végétation? Peut-ètre, mais seulement sur les vignes touffues et insuffisamment éclairées. Ce cas doit ètre plutòt rare.

\section{GONGLUSIONS}

$1^{\circ}$ Le rognage réduit l'importance relative des sommets parasites : effet utile qui se traduit par un accroissement de la production (réduction de la coulure, grossissement des grains de raisin, etc.)

2 - Son ròle va en diminuant et tend vers zéro depuis le débourrement jusqu'à l'arrèt de la végétation. Courbe $\frac{\mathrm{A}}{\mathrm{C}}=\mathrm{B}$.

$3^{\circ}$ Il augmente l'éclairement des grappes : effet utile au point de vue qualité ; indifférent sur la quantité.

4. II réduit la surface foliaire : effet nuisible et pour la qualité et pour la quantité.

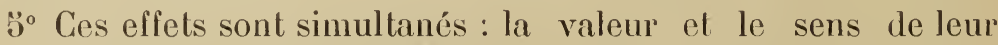
résultante doivent ètre variables.

$6^{\circ}$ Ils sont d'autant moins apparents que les cépages rognés sont meilleurs producteurs (Aramon, etc.) et les rameaux plus recourbés.

$7^{\circ}$ Quand il augmente la production, il diminue la qualité. Il la diminue aussi en prolongeant l'état de vie active de la plante.

$8^{\circ}$ Il agit sur la croissance et la maturation des rameaux et du feuillage comme sur les grappes. 
$9^{\circ}$ En conséquence, il augmente généralement la sensibilité du feuillage restant aux maladies (mildiou, etc.) mais il facilite aussi les moyens de les combattre.

$10^{\circ}$ Peut.ètre hàte-t-il la mise à fruits des bourgeons basilaires?

\section{TECHNIQUE}

La prescription de rogner à 1, 2, 3 et 4 feuilles au-dessus de la dernière grappe, n'a aucun sens. Pratiqué de bonne heure, un rognage à une feuille est d'une efficacité sensiblement nulle ; pratiqué tardivement, il entraine la suppression de presque tout le feuillage. Comment s'étonner, après cela, que des rognages maintenant une mème surface foliacée, donnent si souvent des résultats divergents. ? Ce n'est pas ce qui reste qu'il fautconsidérer, c’est ce qui est enlevé.

Aussi dans nos expériences nous n'avons pas opéré ainsi. Toute discussion des résultats eut été impossible. J'ai préféré, dans tous les cas, supprimer l'extrémité en voie de croissance,la partie restante dépassant toujours les grappes --, c'est-à-dire les $\check{5}, 6$ et $7 \ldots$. mérithalles terminaux.

Dans la pratique, cette opération est faite, quand on désire favoriser la fructification, avant, pendant ou un peu après la floraison, à la main, ou avec une serpette, une faucille ou des cisailles. Les rognages ultérieurs sont faits de la mème manière.

Quelquefois, dans les vignes taillées long, le rognage est pratiqué en deux fois : il porte d'abord sur les rameaux des branches à fruit qui ne doivent pas ètre conservées à la taille suivanle; et plus tard, longtemps après la floraison, sur les rameaux qui doivent ètre utilisés à la taille (Système Guyot). 



\section{LIBRAIRIE COULET ET FILS, ÉDITEURS, MONTPELLIER}

BERNE (A.). - Manuel d'arboriculture fruitière, par A. Berne, jardınier en chef à l'Ecole d'agriculture de Montpellier. 1 vol. in- 8 écu, aver nombreuses illustrations.

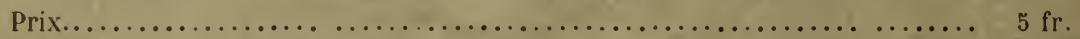

CAZALIS (Fr.). - Traité pratique de l'Art de faire le Vin, par le Docteur Fr. CAzAuss, directeur du Messager agricole, présídent de la Société centrale d'agriculburc de l'Hérault. Deuxième édition, revue et corrigéc. 1. vol. in-8, avec tigures dans le

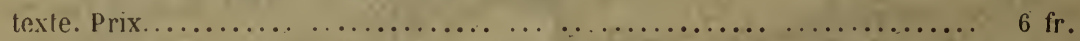

FoFX (G.). - Manuel pratique pour la Reconstitution des vignobles méridionaux, par G. Forx, ancien directeur de l'Ecole d'agriculture de Montpellier, ilspecteur général de la Viticulture. $6^{\mathrm{m}_{0}}$ édition, revue et augmentée. 1 vol. in-8 écu. Prix .................................... $4 \mathrm{fr}$.

NAYET (Valéry). - Les Insečtes de la Vigne et les Moyens de les combattre, par V. MAYET, professeur à l'Ecole d’agriculture de Montpcllier. 1 vol. in-8, avec planches et figures dans le texte Prix...................... $10 \mathrm{fr}$.

MICHAUD et VERMOREL. - Les Engrais de la Vigne, par Mıchaud, chimiste à la Station viticole de Villefranche, et V. Vвrmores, président du Comice agricole de Villefranche. Deuxième édition, revue el augmentée. 1 vol. in-8 écu. Prix. -3 fr. 50

RABATÉ et BERNÈS. - La Chenille fileuse du Prunier, par E. Rabaté, professeur départemental d'agriculture, et E. BernÈs, professeur a'agriculture. 1 brochure in-8, avec planches en couleurs, 1909. Prix................... $1 \mathrm{f}$.

RAVAZ (L.). - Influence des opérations culturales sur la végétation et la production de la Vigne, par L. RAVAz, professeur à l'Ecole d'agriculture de Montpellier. 1 brochure in-8, 1909. Prix......................... $1 \mathrm{fr}$.

RAVAZ ET DEGRLLLY. - La Culture superficielle de la Vigne, par L. RAVAZ et L. DEgrully, professeur's à l'Ecole d'agriculture de Montpellier. 1 vol. in-8, avec figu-

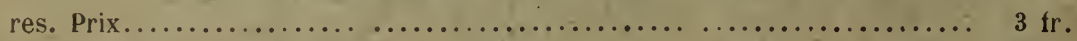

VENTRE (Jules). - Les Levures dans la Vinification. Multiplicalion, Aliments, Sélection, Technique de l'emploi des Levures, par Jules Ventre, docteur ès Sciences, préparateur à l'École d'agriculture de Montpellier. 1 vol. in-12, 1911. Prix. 2 fr. 50

- Le Phosphore dans les Raisins et le Vin. Ses formes, par Jules Ventre, doc-

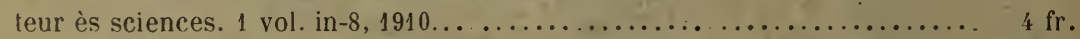

- La Vinification rationnelle des Blancs et des Rosés, par Jules Ventre, docteur ès sciences. 1 brochure in-8. Prix. $1 \mathrm{fr}$. 